\title{
Simultaneous Core Partitions: Parameterizations and Sums
}

\author{
Victor Y. Wang* \\ Department of Mathematics \\ Massachusetts Institute of Technology \\ Massachusetts, U.S.A. \\ vywang@mit.edu
}

Submitted: Aug 7, 2015; Accepted: Dec 29, 2015; Published: Jan 11, 2016

Mathematics Subject Classifications (2010): 05A15, 05A17, 05E10, 05E18

\begin{abstract}
Fix coprime $s, t \geqslant 1$. We re-prove, without Ehrhart reciprocity, a conjecture of Armstrong (recently verified by Johnson) that the finitely many simultaneous $(s, t)$ cores have average size $\frac{1}{24}(s-1)(t-1)(s+t+1)$, and that the subset of self-conjugate cores has the same average (first shown by Chen-Huang-Wang). We similarly prove a recent conjecture of Fayers that the average weighted by an inverse stabilizergiving the "expected size of the $t$-core of a random $s$-core" - is $\frac{1}{24}(s-1)\left(t^{2}-1\right)$. We also prove Fayers' conjecture that the analogous self-conjugate average is the same if $t$ is odd, but instead $\frac{1}{24}(s-1)\left(t^{2}+2\right)$ if $t$ is even. In principle, our explicit methods - or implicit variants thereof - extend to averages of arbitrary powers.

The main new observation is that the stabilizers appearing in Fayers' conjectures have simple formulas in Johnson's $z$-coordinates parameterization of $(s, t)$-cores.

We also observe that the $z$-coordinates extend to parameterize general $t$-cores. As an example application with $t:=s+d$, we count the number of $(s, s+d, s+2 d)$ cores for coprime $s, d \geqslant 1$, verifying a recent conjecture of Amdeberhan and Leven.
\end{abstract}

Keywords: core partition; hook length; beta-set; group action; cyclic shift; Dyck path; rational Catalan number; Motzkin number; numerical semigroup

\section{Introduction}

\subsection{History and motivation}

A partition is an infinite weakly decreasing sequence $\lambda=\left(\lambda_{1}, \lambda_{2}, \ldots\right)$ of nonnegative integers with finite size $|\lambda|:=\lambda_{1}+\lambda_{2}+\cdots$. The Young diagram of $\lambda$ is the set $[\lambda]=$

\footnotetext{
*Supported by NSF grant 1358695 and NSA grant H98230-13-1-0273.
} 


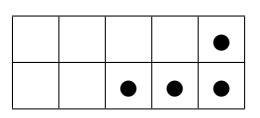

Figure 1: English notation for $\lambda=(5,5,0, \ldots)$. Removing the displayed rim 4-hook (of $(1,3) \in[\lambda])$ leaves the partition $(4,2,0, \ldots)$; removing the remaining rim 4-hook leaves the 4 -core $\lambda^{4}=(1,1,0, \ldots)$.

$\left\{(r, c) \in \mathbb{Z}_{>0}^{2}: c \leqslant \lambda_{r}\right\}$, often visualized as a set of $\#[\lambda]=|\lambda|$ boxes in some orientation. Reflecting $[\lambda]$ about the diagonal $r=c$ gives the diagram of the conjugate partition $\bar{\lambda}=\left(\bar{\lambda}_{1}, \bar{\lambda}_{2}, \ldots\right)$, formally defined by $\bar{\lambda}_{r}:=\#\left\{c \in \mathbb{Z}_{>0}: r \leqslant \lambda_{c}\right\}$ for $r \geqslant 1$; we say $\lambda$ is self-conjugate if $\lambda=\bar{\lambda}$.

A partition $\lambda$ has, associated to each square $(r, c) \in[\lambda]$, a rim hook $\{(i, j) \in[\lambda]: i \geqslant$ $r, j \geqslant c$, and $(i+1, j+1) \notin[\lambda]\}$ of (positive) rim hook length $1+\left(\lambda_{r}-r\right)+\left(\bar{\lambda}_{c}-c\right)$ - the same as the hook length of the usual hook $\{(i, c) \in[\lambda]: i \geqslant r\} \cup\{(r, j) \in[\lambda]: j \geqslant c\}$. Importantly, removing a rim hook of $\lambda$ leaves the Young diagram of a smaller partition. (See Figure 1.) Note that the (finite) set of hook lengths is invariant under conjugation.

When $s$ is a positive integer, we say a partition is an $s$-core if it has no hooks of length $s$, or equivalently no rim s-hooks (rim hooks of length $s$ ); following Fayers [15], we denote by $\mathcal{C}_{s}$ the set of $s$-cores, and by $\mathcal{D}_{s} \subseteq \mathcal{C}_{s}$ the set of self-conjugate $s$-cores. More generally, any partition $\lambda$ has a unique $s$-core $\lambda^{s} \in \mathcal{C}_{s}$, given by repeatedly removing rim $s$-hooks. To prove that this $s$-core operation $\lambda \mapsto \lambda^{s}$ is well-defined, one can use the beta-sets reviewed in Section 2.1, which also show that $\lambda$ is an $s$-core if and only if it has no hook lengths divisible by $s$, unifying two common definitions of $\mathcal{C}_{s}$. These notions are connected to representation theory, symmetric function theory, and number theory (see e.g. $[13,15,19])$.

Going further, many authors (see e.g. $[1,2,3,4,5,6,7,11,13,14,15,16,17,21$, $24,25,27,28,29]$ ) have recently considered the interaction of $s$-cores and $t$-cores (both the partitions and operations), for two positive integers $s, t$. For example, Anderson [5] showed that for coprime $s, t \geqslant 1$, the set $\mathcal{C}_{s} \cap \mathcal{C}_{t}$ of (simultaneous) $(s, t)$-cores has size equal to the number of $(s, t)$-Dyck paths, which Bizley [8] had earlier enumerated-via 'cyclic shifts' - as the 'rational Catalan number' $\frac{1}{s+t}\left(\begin{array}{c}s+t \\ t\end{array}\right) .{ }^{1}$ Ford, Mai, and Sze [17] later showed that for coprime $s, t \geqslant 1$, the set $\mathcal{D}_{s} \cap \mathcal{D}_{t}$ of self-conjugate $(s, t)$-cores has size equal to the lattice path count $\left(\begin{array}{c}\lfloor s / 2\rfloor+\lfloor t / 2\rfloor \\ \lfloor t / 2\rfloor\end{array}\right)$. In a different direction, Olsson [24] showed that the $t$-core of an $s$-core is an $s$-core, hence a simultaneous $(s, t)$-core (as it is a $t$-core by definition).

In this paper, we mainly focus on related conjectures of Armstrong from [6], and Fayers from [15], on certain weighted average sizes of $(s, t)$-cores when $s, t$ are coprime. Chen, Huang, and Wang [11] established Armstrong's self-conjugate conjecture (Theorem 1.2 below) using the Ford-Mai-Sze bijection [17]. Using a poset formulation of Anderson's

\footnotetext{
${ }^{1}$ In fact, $(s, t)$-cores biject to subsets of $\mathbb{Z}_{\geqslant 0}$ that contain 0 and are closed under addition by $s, t$ (e.g. via beta-sets, following negation and suitable translation), which are counted in [18]. Through this bijection, numerical semigroups containing $s, t$ inject into $\mathcal{C}_{s} \cap \mathcal{C}_{t}$.
} 
bijection [5], Stanley and Zanello [27] recursively established Armstrong's general conjecture (Theorem 1.1 below) for the 'Catalan case $t=s+1$ '; Aggarwal [1] generalized their method to the case $t \equiv 1(\bmod s)$. However, it is unclear whether a similar 'Catalan-like' recursive structure holds for other choices of $s, t$. Recently, by different means described below, Johnson [21] fully proved Theorem 1.1, and re-proved Theorem 1.2.

Theorem 1.1 (Armstrong [6, Conjecture 2.6]; Johnson [21, Corollary 3.8]). Fix coprime $s, t \geqslant 1$. Then

$$
\frac{\sum_{\lambda \in \mathcal{C}_{s} \cap \mathcal{C}_{t}}|\lambda|}{\sum_{\lambda \in \mathcal{C}_{s} \cap \mathcal{C}_{t}} 1}=\frac{1}{24}(s-1)(t-1)(s+t+1),
$$

where the sums run over all $(s, t)$-core partitions.

Theorem 1.2 (Armstrong [6, Conjecture 2.6]; Chen-Huang-Wang [11, proof in Section 2]; Johnson [21, Proposition 3.11]). Fix coprime $s, t \geqslant 1$. Then

$$
\frac{\sum_{\lambda \in \mathcal{D}_{s} \cap \mathcal{D}_{t}}|\lambda|}{\sum_{\lambda \in \mathcal{D}_{s} \cap \mathcal{D}_{t}} 1}=\frac{1}{24}(s-1)(t-1)(s+t+1),
$$

where the sums run over all self-conjugate $(s, t)$-core partitions.

Developing Olsson's [24] and his own [13] ideas, Fayers soon after conjectured weighted analogs (Theorems 1.3 and 1.4 below) of Armstrong's conjectures - in some sense giving the "expected size of the $t$-core of a random $s$-core" [15]. In this paper, we carry over explicit versions of Johnson's methods to establish both of Fayers' conjectures, despite the absence of an obvious 'exponential' analog of Ehrhart reciprocity. We also briefly explain, in Remark 8.3, how one could give more implicit or "conceptual" proofs if necessary.

Theorem 1.3 (Fayers [15, Conjecture 3.1]). Fix coprime $s, t \geqslant 1$. Then

$$
\frac{\sum_{\lambda \in \mathcal{C}_{s} \cap \mathcal{C}_{t}}\left|\operatorname{Stab}_{G_{s, t}}(\lambda)\right|^{-1} \cdot|\lambda|}{\sum_{\lambda \in \mathcal{C}_{s} \cap \mathcal{C}_{t}}\left|\operatorname{Stab}_{G_{s, t}}(\lambda)\right|^{-1} \cdot 1}=\frac{1}{24}(s-1)\left(t^{2}-1\right)
$$

where the sums run over all $(s, t)$-core partitions, and the stabilizers are defined in terms of Fayers' 'level t' group action on $\mathcal{C}_{s}[13,15]$ reviewed in Definition 3.7.

Theorem 1.4 (Fayers [15, Conjecture 4.5]). Fix coprime $s, t \geqslant 1$. Then

$$
\frac{\sum_{\lambda \in \mathcal{D}_{s} \cap \mathcal{D}_{t}}\left|\operatorname{Stab}_{H_{s, t}}(\lambda)\right|^{-1} \cdot|\lambda|}{\sum_{\lambda \in \mathcal{D}_{s} \cap \mathcal{D}_{t}}\left|\operatorname{Stab}_{H_{s, t}}(\lambda)\right|^{-1} \cdot 1}=\left\{\begin{array}{lll}
\frac{1}{24}(s-1)\left(t^{2}-1\right) & \text { if } t \equiv 1 & (\bmod 2) \\
\frac{1}{24}(s-1)\left(t^{2}+2\right) & \text { if } t \equiv 0 & (\bmod 2)
\end{array},\right.
$$

where the sums run over all self-conjugate $(s, t)$-core partitions, and the stabilizers are defined in terms of Fayers' 'level t' group action on $\mathcal{D}_{s}$ [15] reviewed in Definition 6.6.

Remark 1.5. As Fayers notes in [15], the orbits of $G_{s, t}$ and $H_{s, t}$ are infinite, so one weights by the inverses of the finite stabilizers instead. However, by considering finite quotients acting on certain finite subsets of $\mathcal{C}_{s}$ and $\mathcal{D}_{s}$, he also gives the weighted averages finite probabilistic interpretations that agree exactly, not just asymptotically, with the original averages. 
Johnson's $z$-coordinates parameterization of $(s, t)$-cores, and our modest extension to general $t$-cores (see Proposition 4.2 for general cores and in Proposition 6.11 for the selfconjugate specialization) - which depends on a choice of $s \geqslant 1$ coprime to $t$-plays a key role in our paper, which rests upon his cyclic shifts argument for general cores. However, whereas Johnson finishes by 'weighted Ehrhart reciprocity' (see [9] for an introduction to 'un-weighted' Ehrhart theory), we will stick to flexible direct tools - and implicit variants thereof - which can in principle evaluate sums of arbitrary powers of the partition sizes (see Remark 8.6).

The main new observation is that the stabilizer sizes appearing in Fayers' conjectures (Theorems 1.3 and 1.4) have symmetric (or almost symmetric) formulas in the $z$-coordinates, which simply re-index the restricted counts $\left|\mathcal{S}_{s}(\lambda) \cap(j+t \mathbb{Z})\right|$ of elements in Fayers' $s$-sets $\mathcal{S}_{s}(\lambda)$ (from $[13,15]$ ). In fact, we first prove (in Propositions 3.4 and 3.6) that the sets $\mathcal{S}_{s}(\lambda) \cap(j+t \mathbb{Z})$ underlie the tools allowing us, in Fayers' words [13], to "[compare] the $t$-cores of different $s$-cores" in the first place-thus illustrating the significance of $z$-coordinates.

As an application of the cyclic shifts in the (extended) $z$-coordinates, we also parameterize and then enumerate the simultaneous $(m, m+d, m+2 d)$-cores for coprime $m, d \geqslant 1$, verifying the following recent conjecture of Amdeberhan and Leven [4] in two different ways.

Theorem 1.6 (Amdeberhan-Leven, [4, Conjecture 3.1]). Fix coprime $m, d \geqslant 1$. Then

$$
\sum_{\lambda \in \mathcal{C}_{m} \cap \mathcal{C}_{m+d} \cap \mathcal{C}_{m+2 d}} 1=\frac{1}{m+d} \sum_{i=0}^{\lfloor m / 2\rfloor}\left(\begin{array}{c}
m+d \\
i, i+d, m-2 i
\end{array}\right) .
$$

([4] also gives an equivalent expression using $\frac{1}{m+d}\left(\begin{array}{c}m+d \\ i, i+d, m-2 i\end{array}\right)=\left(\begin{array}{c}m+d-1 \\ 2 i+d-1\end{array}\right)\left(\begin{array}{c}2 i+d \\ i\end{array}\right) \frac{1}{2 i+d}$.)

Remark 1.7. Following the recursive method of Stanley and Zanello [27], Amdeberhan and Leven [4] (and independently, Yang, Zhong, and Zhou [29]) proved the $d=1$ case via Motzkin number interpretations. Both papers also proved other facts about $(m, m+$ $1, m+2)$-cores.

Remark 1.8. A few days after the arXiv postings of $\mathrm{v} 2$ of the present paper and $\mathrm{v} 1$ of [2], Paul Johnson informed us (via private correspondence) that he had independently found our asymmetric proof (in Section 7) of Theorem 1.6. In fact, he proved the slightly stronger result that the term $\frac{1}{m+d}\left(\begin{array}{c}m+d \\ i, i+d, m-2 i\end{array}\right)$ counts the number of $(m, m+d, m+2 d)$-cores with exactly $i$ hooks of length $d$; see the end of Remark 3.5 for a brief explanation.

\subsection{Outline of paper}

In Section 2, we review the relevant definitions, terminology, and basic results about $s$ core partitions and the $s$-core operation on partitions, mostly from Fayers $[13,15]$ and Johnson [21]. Section 3 provides the fundamental results on $t$-cores of $s$-cores, giving the background needed to state Theorems 1.3 and 1.4 (Fayers' conjectures). We isolate 


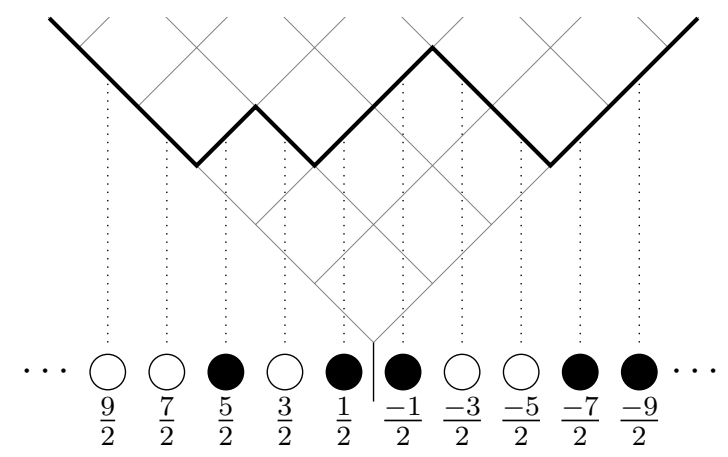

Figure 2: Russian notation for $\lambda=(3,2,2,0, \ldots)$, from Johnson's arXiv source [21]. (As pointed out by an anonymous expert, the idea itself has been used earlier by Okounkov and Reshetikhin.) The infinite rim boundary is thickened. A rim edge has a filled circle below if and only if the edge slopes upwards. By convention, our labeling increases from right to left (opposite the usual Cartesian $x$-axis).

our main new observation as Proposition 3.4, which first gives a cleaner proof of a key proposition from [13], and later features in our stabilizer computations.

Section 4 describes the relevant computations for Section 5 (on general cores): we compute the sizes of the stabilizers appearing in Theorem 1.3 and express $s$-set data, stabilizer sizes, and partition sizes in Johnson's z-coordinates. We also extend the $z$ coordinates, and review the cyclic shifts used to compute $z$-coordinate sums of cyclic functions, such as the stabilizer and partition sizes.

Section 5 presents the main results of the paper, namely explicit proofs of Theorems 1.1 and 1.3 (general conjectures). Section 6 presents self-conjugate analogs of the general analysis, building up to explicit proofs of Theorems 1.2 and 1.4 (self-conjugate conjectures).

Section 7 uses cyclic shifts in the extended z-coordinates from Section 4.2 to quickly prove Theorem 1.6. Section 8 discusses possibilities for future work, including the question of calculating sums of higher powers (or moments) of the $(s, t)$-core partition sizes.

\section{Background: $s$-core partitions and operation}

Experts can quickly skim this section for the notation used in our paper (as the literature seems to have many different conventions), particularly the framework of beta-sets (Section 2.1) for studying hooks, and two parameterizations of $s$-cores: Johnson's c-coordinates (Section 2.2) from [21], and Fayers' $s$-sets and a-coordinates (Section 2.4) from [13, 15].

\section{$2.1 \quad$ Beta-sets}

To each partition one naturally associates a beta-set illuminating the hook length structure. It is often easier to work with beta-sets than with partitions themselves. 
Definition 2.1 ([21, Section 2.2]). For any (infinite weakly decreasing) partition $\lambda=$ $\left(\lambda_{1}, \lambda_{2}, \ldots\right)$, let $B^{\lambda} \subset \mathbb{Z}$ denote the (infinite) beta-set of beta-values, i.e. integers $x$ such that the the rim edge (with midpoint) at position $x+\frac{1}{2}$ slopes upwards in Figure 2.

Remark 2.2. Most sources (e.g. $[13,15,20])$ instead define $B^{\lambda}:=\left\{\lambda_{i}-i\right\}_{i \geqslant 1}$. The definitions are equivalent by a simple coordinate geometry argument. Some sources, notably Anderson [5] (and the poset method users [27, 1, 4, 3], by extension) and Vandehey [28], instead distinguish the finite set $\left(B^{\lambda}+r\right) \cap \mathbb{Z}_{>0}=\left\{\left(\lambda_{1}-1\right)+r, \ldots,\left(\lambda_{r}-r\right)+r\right\}$ of first column hook lengths, where $r$ denotes the length of $\lambda$, defined so that $\lambda_{1} \geqslant \cdots \geqslant$ $\lambda_{r}>0=\lambda_{r+1}=\cdots$. This perspective works particularly well for the study of maximal cores (see e.g. [28] for $(s, t)$-cores and [3] for $(a, b, c)$-cores).

Proposition 2.3 (Robinson [26, 2.8]; Johnson [21, Section 2.3.1]). A rim s-hook is parameterized (uniquely) by an element $x \in B^{\lambda} \backslash\left(B^{\lambda}+s\right)$. In particular, a partition $\lambda$ is an $s$-core if and only if $B^{\lambda}-s \subseteq B^{\lambda}$.

\subsection{Johnson's signed 'charge' measure $c$}

In light of Proposition 2.3, one would like to have a clean description of possible beta-sets. The basic tool for this is charge, which also parameterizes $s$-cores in Corollary 2.7.

Definition 2.4 (c.f. [21, Definition 2.1 and Section 2.2]; [19]; [12]). Call a set $S \subseteq \mathbb{Z}$ good if $S \cap \mathbb{Z}_{>0}$ and $\mathbb{Z}_{<0} \backslash S$ are both finite. For good $S$, define the signed $s$-charge measure

$c_{s, i}(S):=\#\left[(-1-i+s \mathbb{Z}) \cap(\mathbb{Z} \backslash S) \cap\left\{x+\frac{1}{2}<0\right\}\right]-\#\left[(-1-i+s \mathbb{Z}) \cap S \cap\left\{x+\frac{1}{2}>0\right\}\right]$

for any $s \geqslant 1$ and $i \in \mathbb{Z} / s \mathbb{Z}$. Then $\sum_{i \in \mathbb{Z} / s \mathbb{Z}} c_{s, i}(S)=c_{1,0}(S)$ is the total charge of $S$.

For any partition $\lambda$, we may define $c_{s, i}(\lambda):=c_{s, i}\left(B^{\lambda}\right)$, since $B^{\lambda}$ is good.

Remark 2.5. We will not use the notions of electron, positron, and Maya diagram from Johnson's exposition.

The basic importance of charge is given by the following charge condition.

Proposition 2.6 ([21, Section 2.2]). Fix $s \geqslant 1$. Via beta-sets, partitions are parameterized (uniquely) by good sets $S$ (as defined in Definition 2.4) with $\sum_{i \in \mathbb{Z} / s \mathbb{Z}} c_{s, i}(S)$ equal to 0 .

Combining the $s$-core criterion $B^{\lambda}-s \subseteq B^{\lambda}$ (from Proposition 2.3) with the preceding zero charge invariant yields the following simple $c$-coordinates parameterization of $s$-cores. For the conversion to Fayers' a-coordinates, see Section 2.4.

Corollary 2.7 (Johnson [21, Lemma 2.8]; Garvan-Kim-Stanton [19, Bijection 2]). Fix $s \geqslant 1$. The $s$-cores are parameterized (uniquely) by s-tuples $\left(c_{s, i}\right)_{i \in \mathbb{Z} / s \mathbb{Z}} \in \mathbb{Z}^{s}$ summing to 0 . 


\subsection{The $s$-core operation is well-defined}

Beta-sets not only parameterize rim $s$-hooks (Proposition 2.3), but also conveniently describe the removal of rim $s$-hooks, as follows.

Proposition 2.8 ([21, Section 2.3.1]). The removal (from a partition $\lambda$ ) of a rim s-hook, say parameterized by $x \in B^{\lambda} \backslash\left(B^{\lambda}+s\right)$, corresponds to an s-push: replacing the element $x$ of $B^{\lambda}$ by $x-s$. The process also preserves the charge $s$-tuple $\left(c_{s, i}\right)_{i \in \mathbb{Z} / s \mathbb{Z}}$.

Definition 2.9 (c.f. [21, Section 2.3. Abaci.]). The s-push of a good set $S$ is the welldefined result of repeatedly applying the s-pushes defined in Proposition 2.8. Explicitly, the $s$-push of $S$ can be described as follows:

- Fix a residue class $i+s \mathbb{Z}$; then $S \cap\{i+s \mathbb{Z}\}$ takes the form $\{\ldots, x-2 s, x-s, x, x+$ $\left.\alpha_{1} s, \ldots, x+\alpha_{k} s\right\}$ for any sufficiently small $x \in S \cap\{i+s \mathbb{Z}\}$.

- Then the $s$-push of $S$, restricted to the residue class $i+s \mathbb{Z}$, is $\{\ldots, x-2 s, x-$ $s, x, x+s, \ldots, x+k s\}$.

Repeatedly applying Proposition 2.8 shows that the $s$-core operation is well-defined.

Proposition 2.10 (c.f. [21, Section 2.3.1]). Every partition $\lambda$ has a unique s-core, denoted by $\lambda^{s} \in \mathcal{C}_{s}$. Its beta-set $B^{\lambda^{s}}$ is the s-push of the original beta-set $B^{\lambda}$, so $c_{s, i}(\lambda)=c_{s, i}\left(\lambda^{s}\right)$ for all $i \in \mathbb{Z} / s \mathbb{Z}$.

\subsection{Fayers' $s$-sets versus Johnson's $c$-coordinates}

For any $s$-core $\lambda$, let $a_{s, i}(\lambda):=s+\max \left[B^{\lambda} \cap(i+s \mathbb{Z})\right]$ for each $i \in \mathbb{Z}$. But Definition 2.4 and Corollary 2.7 give $c_{s,-1-i}(\lambda)$ in terms of $B^{\lambda} \cap(i+s \mathbb{Z})$. Comparing the two descriptions gives $a_{s, i}=i-s c_{s,-1-i}$ for $0 \leqslant i \leqslant s-1$. In particular, $\sum_{i \in \mathbb{Z} / s \mathbb{Z}} c_{s, i}=0$ (Proposition 2.6) is equivalent to $\sum_{i \in \mathbb{Z} / s \mathbb{Z}} a_{s, i}=\left(\begin{array}{l}s \\ 2\end{array}\right)$.

Proposition 2.11 (Fayers [13]; [15, Section 3.3]). Fix $s \geqslant 1$. The s-cores are parameterized (uniquely) by $s$-sets $\mathcal{S}_{s}=\left\{a_{s, i}\right\}_{i \in \mathbb{Z} / s \mathbb{Z}}$ of a-coordinates summing to $\left(\begin{array}{l}s \\ 2\end{array}\right)$ with $a_{s, i} \equiv i$ $(\bmod s)$ for all $i \in \mathbb{Z}$. Explicitly, $\mathcal{S}_{s}(\lambda):=\left(B^{\lambda}+s\right) \backslash B^{\lambda}$ for $\lambda \in \mathcal{C}_{s}$.

Remark 2.12. In view of $c_{s, i}(\lambda)=c_{s, i}\left(\lambda^{s}\right)$ from Proposition 2.10, it would be meaningful to define $a_{s, i}(\lambda):=a_{s, i}\left(\lambda^{s}\right)$ for any $\lambda$. However, we will only speak of $s$-sets and $a$-coordinates of $s$-cores. Charge will suffice for our greater needs in Proposition 6.2 and Corollary 6.3.

\section{Background: $t$-cores of $s$-cores}

In this section, we go through the fundamental results on $t$-cores of $s$-cores. In particular, we isolate the crucial Proposition 3.4, meanwhile giving a cleaner proof of a key result of Fayers (see Proposition 3.6). This motivates Fayers' 'level $t$ action on $s$-cores' (Definition 3.7), defining the stabilizers in Theorem 1.3 (Fayers' general conjecture). 


\subsection{When is a $t$-core an $s$-core?}

The following simple criterion parameterizes $\mathcal{C}_{s} \cap \mathcal{C}_{t}$ in $a_{t}$-coordinates. It implicitly appears throughout this paper and elsewhere.

Lemma 3.1 (Johnson [21, Lemma 3.1]; re-worded by Fayers [15, Lemma 3.8]). In Fayers' a-coordinates (see Proposition 2.11), the set $\mathcal{C}_{s} \cap \mathcal{C}_{t}$ of $s$-cores within the affine lattice $\mathcal{C}_{t}$ of $t$-cores is defined by the system of inequalities $a_{t, i} \geqslant a_{t, i+s}-s$ for $i \in \mathbb{Z} / t \mathbb{Z}$.

\subsection{Comparing $t$-cores of different $s$-cores}

Propositions 3.2, 3.4, and 3.6 below are the key conceptual inputs for comparing $t$-cores of various $s$-cores. We start by extending Olsson's theorem [24], following Fayers [13].

Proposition 3.2 (c.f. [13, proof of Proposition 4.1]). Fix any $s, t \geqslant 1$ and $\lambda \in \mathcal{C}_{s}$. Then $\lambda^{t} \in \mathcal{C}_{s} \cap \mathcal{C}_{t}$, and furthermore $\mathcal{S}_{s}\left(\lambda^{t}\right) \equiv \mathcal{S}_{s}(\lambda)$ ( $\left.\bmod t\right)$ (viewed as multisets of residues).

Proof sketch. Given $\lambda \in \mathcal{C}_{s}$, Fayers inductively constructs a sequence of $s$-core partitions $\lambda^{(0)}, \ldots, \lambda^{(-m)}$ (with $m \geqslant 0$ ) such that $\lambda^{(0)}=\lambda$, the term $\lambda^{(-i-1)}$ is an $s$-core obtained from $\lambda^{(-i)}$ by removing a certain sequence of rim $t$-hooks, and $\lambda^{(-m)} \in \mathcal{C}_{s} \cap \mathcal{C}_{t}$. $\mathrm{He}$ shows, under this construction, that $\mathcal{S}_{s}\left(\lambda^{(-i-1)}\right) \equiv \mathcal{S}_{s}\left(\lambda^{(-i)}\right)(\bmod t)$ for $0 \leqslant i<m$, so $\mathcal{S}_{s}\left(\lambda^{(-m)}\right) \equiv \mathcal{S}_{s}(\lambda)(\bmod t)$. But $\lambda^{(-m)}=\lambda^{t}$ by uniqueness of the $t$-core (Proposition $2.10)$.

Remark 3.3. One can avoid induction by first comparing $\left(B^{\lambda}-s\right) \cap(j+t \mathbb{Z})$ with $B^{\lambda} \cap(j+t \mathbb{Z})$ as $j \in \mathbb{Z}$ varies, and then (in view of Proposition 2.10) the $t$-pushes $\left(B^{\lambda^{t}}-s\right) \cap(j+t \mathbb{Z})$ and $B^{\lambda^{t}} \cap(j+t \mathbb{Z})$.

We isolate our main new observation as the following proposition, which first gives a cleaner proof of a key result from [13] (see Proposition 3.6), and later features in our stabilizer computations, namely Propositions 4.1 (general case) and 6.12 (self-conjugate analog).

Proposition 3.4. Fix any $s, t \geqslant 1$ and $\lambda \in \mathcal{C}_{s}$. Then

$$
\left|\left[\mathcal{S}_{s}(\lambda)-s\right] \cap(j+t \mathbb{Z})\right|=\frac{1}{t}\left(a_{t, j}\left(\lambda^{t}\right)-\left[a_{t, j+s}\left(\lambda^{t}\right)-s\right]\right) .
$$

Proof. Proposition 3.2 says $\lambda^{t} \in \mathcal{C}_{s}$ and $\mathcal{S}_{s}(\lambda) \equiv \mathcal{S}_{s}\left(\lambda^{t}\right)(\bmod t)$. Thus it suffices to prove the result with $\lambda$ replaced by its $t$-core $\lambda^{t}$.

In other words, we may without loss of generality assume $\lambda \in \mathcal{C}_{s} \cap \mathcal{C}_{t}$. Recall the $t$-core criterion $B^{\lambda}-t \subseteq B^{\lambda}$ from Proposition 2.3. Then by definition of $a_{t, j} \equiv j(\bmod t)$ and $a_{t, j+s} \equiv j+s(\bmod t)$, we have

$$
\begin{aligned}
B^{\lambda} \cap(j+t \mathbb{Z}) & =\left\{\ldots, a_{t, j}-2 t, a_{t, j}-t\right\} \\
\left(B^{\lambda}-s\right) \cap(j+t \mathbb{Z}) & =\left\{\ldots,\left[a_{t, j+s}-s\right]-2 t,\left[a_{t, j+s}-s\right]-t\right\} .
\end{aligned}
$$

However, Proposition 2.11 gives $\mathcal{S}_{s}(\lambda)-s=B^{\lambda} \backslash\left(B^{\lambda}-s\right)$ for the $s$-core $\lambda$ (with the criterion $B^{\lambda}-s \subseteq B^{\lambda}$ implicit $)$, so $\left[\mathcal{S}_{s}(\lambda)-s\right] \cap(j+t \mathbb{Z})$ is the difference- $t$ arithmetic progression $\left\{a_{t, j+s}-s, \ldots, a_{t, j}-t\right\}$ of nonnegative length $\frac{1}{t}\left(a_{t, j}-\left[a_{t, j+s}-s\right]\right)$. 
Remark 3.5. Compare with both the statement and proof of Lemma 3.1, which can be rephrased in terms of the quantities $\omega:=\frac{1}{t}\left(a_{t, j}-\left[a_{t, j+s}-s\right]\right)$. When $s, t$ are coprime, these re-index in Corollary 4.4 to form Johnson's $z$-coordinates for $(s, t)$-cores $\lambda$ (as vaguely mentioned in the introduction).

Furthermore, for arbitrary t-cores $\lambda$, the quantity $\omega$ is meaningful not only when $\omega \geqslant 0$ (in which case $\omega=\left|\left(B^{\lambda} \backslash\left(B^{\lambda}-s\right)\right) \cap(j+t \mathbb{Z})\right|$ ), but also when $\omega \leqslant 0$ (in which case $-\omega \geqslant 0$ counts the size of $\left(\left(B^{\lambda}-s\right) \backslash B^{\lambda}\right) \cap(j+t \mathbb{Z})$, i.e. the number of rim $s$ hooks coming - via Proposition 2.3-from beta-values $x+s \in B^{\lambda} \cap(j+s+t \mathbb{Z})$ with $\left.(x+s)-s \notin B^{\lambda}\right)$. The latter observation is essentially due to Paul Johnson (via private correspondence), and gives combinatorial significance to our modest extension of his $z$ coordinates (see the second halves of Propositions 4.2 and 6.11).

Proposition 3.4 cleanly proves a result of Fayers "crucial" for "comparing the $t$-cores of different $s$-cores" [13].

Proposition 3.6 (Extension of [13, Proposition 4.1]). Fix coprime $s, t \geqslant 1$. Then the $t$-core $\lambda^{t}$ of an s-core $\lambda$ is uniquely determined by the multiset of modulo $t$ residues $\mathcal{S}_{s}(\lambda)$ $(\bmod t)$. Combined with Proposition 3.2, we conclude that $\lambda, \mu \in \mathcal{C}_{s}$ have the same $t$-core if and only if the multisets of modulo $t$ residues $\mathcal{S}_{s}(\lambda), \mathcal{S}_{s}(\mu)(\bmod t)$ are congruent.

Proof. Fix $\lambda \in \mathcal{C}_{s}$, so Proposition 3.4 gives $\left|\mathcal{S}_{s}(\lambda) \cap(j+s+t \mathbb{Z})\right|=\frac{1}{t}\left(a_{t, j}\left(\lambda^{t}\right)-\left[a_{t, j+s}\left(\lambda^{t}\right)-\right.\right.$ $s])$. Since $s, t$ are coprime, it follows that $\mathcal{S}_{s}(\lambda)(\bmod t)$ determines $\mathcal{S}_{t}\left(\lambda^{t}\right)=\left\{a_{t, j}\left(\lambda^{t}\right)\right\}$ up to translation. The sum condition $\sum_{j \in \mathbb{Z} / t \mathbb{Z}} a_{t, j}\left(\lambda^{t}\right)=\left(\begin{array}{l}t \\ 2\end{array}\right)$ (from Proposition 2.11) then singles out a unique translate equal to $\mathcal{S}_{t}\left(\lambda^{t}\right)$, which corresponds under Proposition 2.11 to a unique $t$-core $\lambda^{t}$.

\subsection{Level $t$ action and statement of Fayers' general conjecture}

Proposition 3.6 motivates the following group action on $\mathcal{C}_{s}$, for which Corollary 3.11 will hold almost by definition.

Definition 3.7 (c.f. Fayers [13, Section 3.2]; [15, Section 3.1]). Fix coprime $s, t \geqslant 1$. Let $G_{s, t}$ be the set of permutations $f: \mathbb{Z} \rightarrow \mathbb{Z}$ such that

- $f$ is $s$-periodic, i.e. $f(m+s)=f(m)+s$ for all $m$;

- $f$ satisfies the sum-invariance condition $\sum_{i=0}^{s-1} f(i)=\left(\begin{array}{c}s \\ 2\end{array}\right)$;

- $f$ preserves residues modulo $t$, i.e. $f(m) \equiv m(\bmod t)$ for all $m$.

It is easy to check that $G_{s, t}$ has a group structure, and that it acts in the obvious way on the set of $s$-sets $\mathcal{S}_{s}(\lambda)$ (of $s$-cores $\lambda$ ), or equivalently on beta-sets $B^{\lambda}$ (of $s$-cores $\lambda$ ). This induces an action on $\mathcal{C}_{s}$, via Proposition 2.11 .

Remark 3.8 (Different but equivalent definitions). We have not given Fayers' actual definition of the 'level $t$ action of the $s$-affine symmetric group' (based on the $t=1$ case from [23]), but rather one equivalent by [15, Proposition 3.5], and easier to work with for our purposes. 
Remark 3.9 (Natural action?). It is not hard to show that a permutation $f: \mathbb{Z} \rightarrow \mathbb{Z}$ preserves the set of beta-sets of $s$-cores if and only if it satisfies the explicit $s$-periodicity and sum-invariance conditions.

In practice one often encounters group elements by their restrictions to $s$-sets.

Proposition 3.10 ([15, Corollary 3.6]). Let $\lambda, \mu$ be s-cores. Suppose $\phi$ a set bijection $\phi: \mathcal{S}_{s}(\lambda) \rightarrow \mathcal{S}_{s}(\mu)$ that preserves residue classes modulo $t$. Then $\phi$ uniquely extends to an element $f \in G_{s, t}$ (under Definition 3.7).

Corollary 3.11 ([13, Proposition 4.2 and Corollary 4.5]). Fix coprime $s, t \geqslant 1$. Then $\lambda, \mu \in \mathcal{C}_{s}$ lie in the same $G_{s, t}$-orbit if and only if $\lambda^{t}=\mu^{t}$. In other words, each $G_{s, t}$-orbit of $\mathcal{C}_{s}$ contains a unique $t$-core (hence an $(s, t)$-core), and any $\lambda \in \mathcal{C}_{s}$ lies in $G_{s, t} \lambda^{t}$.

Sketch of more direct proof. Fix $s$-cores $\lambda, \mu$. Proposition 3.6 followed by Proposition 3.10 gives equivalence of $\lambda^{t}=\mu^{t}$ and $\mu \in G_{s, t} \lambda$. The re-phrasing follows by specializing to $\mu:=\lambda^{t}$ (where $\lambda^{t} \in \mathcal{C}_{s}$ follows from Proposition 3.2).

Definition 3.7 and Corollary 3.11 provide the background and context for Theorem 1.3 (stated in the introduction).

\section{Key inputs for computation}

In this section, we describe all the computational methods and results used to compute the sums in Theorems 1.1 and 1.3. First we compute the sizes of the stabilizers appearing in Theorem 1.3. Section 4.2 compares Fayers' $a$-coordinates with a modest extension of Johnson's z-coordinates. In Section 4.4 we give an explicit formula for the size of a $t$-core, and in Section 4.3 we explain the standard cyclic shifts used to compute $z$-coordinate sums of cyclic functions, such as the stabilizer and partition sizes.

\subsection{Size of the stabilizer of an $s$-core}

Most of the proof ideas for Theorem 1.3 come from Johnson [21] and Fayers [13, 15]. The key new observation is the following computational simplification of Fayers' formula for the size of the stabilizer of an $s$-core, based on Proposition 3.4, which will simplify even further once we translate to Johnson's $z$-coordinates (see Corollary 4.4).

Proposition 4.1 (c.f. [15, Proposition 3.7]). Fix coprime $s, t \geqslant 1$, and $\lambda \in \mathcal{C}_{s}$. Then with $G_{s, t}$ from Definition 3.7, $\left|\operatorname{Stab}_{G_{s, t}}(\lambda)\right|$ equals $\prod_{j \in \mathbb{Z} / t \mathbb{Z}}\left[\frac{1}{t}\left(a_{t, j}\left(\lambda^{t}\right)-\left[a_{t, j+s}\left(\lambda^{t}\right)-s\right]\right)\right]$ !.

Proof. Using Proposition 3.10, Fayers showed in [15, Proposition 3.7] that $\operatorname{Stab}_{G_{s, t}}(\lambda)$ (under a different but equivalent definition of $G_{s, t}$; see Remark 3.8) has size

$$
\prod_{j \in \mathbb{Z} / t \mathbb{Z}}\left|\mathcal{S}_{s}(\lambda) \cap(j+t \mathbb{Z})\right| !=\prod_{j \in \mathbb{Z} / t \mathbb{Z}}\left|\left[\mathcal{S}_{s}(\lambda)-s\right] \cap(j+t \mathbb{Z})\right| !
$$

Substituting in Proposition 3.4 gives the result. 


\subsection{Johnson's $z$-coordinates versus Fayers' $t$-sets}

As reflected by the simple translation Corollary 4.4, Proposition 4.1 and Remark 3.5 provide one source of motivation for the following choice of coordinates. We not only review Johnson's parameterization of $(s, t)$-cores, but also extend it to arbitrary $t$-cores, given a parameter $s \geqslant 1$ coprime to $t$.

Proposition 4.2 (c.f. [21, Lemma 3.5]: Johnson's $\boldsymbol{z}$-coordinates versus Fayers' $\boldsymbol{t}$-sets). Fix coprime $s, t \geqslant 1$. The set $\mathcal{C}_{s} \cap \mathcal{C}_{t}$ of $(s, t)$-cores (viewed as $s$-cores within the set of t-cores) is parameterized by either of the sets $A_{t}(s), \mathrm{TD}_{t}(s)$, described as follows.

- By Lemma 3.1, the set of $t$-sets $\mathcal{S}_{t}(\lambda)$ of $(s, t)$-cores $\lambda$, i.e. the set of points $A_{t}(s)=\left\{\left(a_{t, i}\right)_{i \in \mathbb{Z} / t \mathbb{Z}\}}\right.$ defined by the inequalities $a_{t, i} \geqslant a_{t, i+s}-s$, the sum condition $\sum_{i \in \mathbb{Z} / t \mathbb{Z}} a_{t, i}=\left(\begin{array}{l}t \\ 2\end{array}\right)$, and congruence conditions $a_{t, i} \equiv i(\bmod t)$.

- Johnson's trivial determinant representations set $\operatorname{TD}_{t}(s)=\left\{\left(z_{t, i}\right)_{i \in \mathbb{Z} / t \mathbb{Z}}\right\}$ defined by the inequalities $z_{t, i} \geqslant 0$, the sum condition $\sum_{i \in \mathbb{Z} / t \mathbb{Z}} z_{t, i}=s$, and congruence conditions $z_{t, i} \equiv 0(\bmod 1)$ and $\sum_{i \in \mathbb{Z} / t \mathbb{Z}} i z_{t, i} \equiv 0(\bmod t)$.

An isomorphism (also preserving the ambient linear and simplex structures) is given by the invertible affine change of variables $z_{t, j}:=\frac{1}{t}\left(a_{t, s j+k}-\left[a_{t, s(j+1)+k}-s\right]\right)$, for $j \in \mathbb{Z} / t \mathbb{Z}$, where $k:=\frac{1}{2}(s+1)(t-1) \in \mathbb{Z}$. The inverse map can be described by $a_{t, k+\ell s}-\frac{t-1}{2}=$ $\sum_{j=0}^{t-1}\left(\frac{t-1}{2}-j\right) z_{t, j+\ell}$, for $\ell \in \mathbb{Z} / t \mathbb{Z}$.

Under the same affine change of variables, the larger set $\mathcal{C}_{t}$ of $t$-cores is parameterized by either of the following sets.

- By Proposition 2.11, the set of $t$-sets $\mathcal{S}_{t}(\lambda)$ of $t$-cores $\lambda$, i.e. the set of points $\mathcal{C}_{t}=\left\{\left(a_{t, i}\right)_{i \in \mathbb{Z} / t \mathbb{Z}}\right\}$ defined by the sum condition $\sum_{i \in \mathbb{Z} / t \mathbb{Z}} a_{t, i}=\left(\begin{array}{l}t \\ 2\end{array}\right)$, and congruence conditions $a_{t, i} \equiv i(\bmod t)$.

- In z-coordinates, the set of points $\mathcal{C}_{t}=\left\{\left(z_{t, i}\right)_{i \in \mathbb{Z} / t \mathbb{Z}\}}\right.$ defined by the sum condition $\sum_{i \in \mathbb{Z} / t \mathbb{Z}} z_{t, i}=s$, and congruence conditions $z_{t, i} \equiv 0(\bmod 1)$ and $\sum_{i \in \mathbb{Z} / t \mathbb{Z}} i z_{t, i} \equiv 0$ $(\bmod t)$.

Remark 4.3. Although it will only matter for the asymmetric Theorem 1.3, not the symmetric Theorem 1.1, we use, in Johnson's notation, the parameterization $\operatorname{TD}_{t}(s)$, instead of $\operatorname{TD}_{s}(t)$ as Johnson might for Armstrong's conjectures [21].

Before proving the result, we first translate $s$-set and stabilizer data to $z$-coordinates.

Corollary 4.4. Fix coprime $s, t \geqslant 1$. Fix $\lambda \in \mathcal{C}_{s}$. The $z$-coordinates parameterizing the $(s, t)$-core $\lambda^{t}$ are defined by the affine change of variables

$$
\underbrace{z_{t, j}\left(\lambda^{t}\right):=\frac{1}{t}\left(a_{t, s j+k}\left(\lambda^{t}\right)-\left[a_{t, s(j+1)+k}\left(\lambda^{t}\right)-s\right]\right)}_{\text {as defined in Proposition 4.2 }} \underbrace{=\left|\left[\mathcal{S}_{s}(\lambda)-s\right] \cap(s j+k+t \mathbb{Z})\right|}_{\text {by Proposition } 3.4},
$$


where $k$ is the constant $\frac{1}{2}(s+1)(t-1)$. Furthermore, Proposition 4.1 translates to

$$
\begin{aligned}
\left|\operatorname{Stab}_{G_{s, t}}(\lambda)\right| & =\prod_{j \in \mathbb{Z} / t \mathbb{Z}}\left(\frac{a_{t, j}\left(\lambda^{t}\right)-\left[a_{t, j+s}\left(\lambda^{t}\right)-s\right]}{t}\right) ! \\
& =\underbrace{\prod_{j \in \mathbb{Z} / t \mathbb{Z}}\left(\frac{a_{t, s j+k}\left(\lambda^{t}\right)-\left[a_{t, s(j+1)+k}\left(\lambda^{t}\right)-s\right]}{t}\right) !}_{\text {since } j+t \mathbb{Z} \mapsto s j+k+t \mathbb{Z} \text { is bijective }}=\prod_{j \in \mathbb{Z} / t \mathbb{Z}} z_{t, j}\left(\lambda^{t}\right) ! .
\end{aligned}
$$

Proof of a-versus-z isomorphism in Proposition 4.2. Let $\phi$ be the affine map sending a

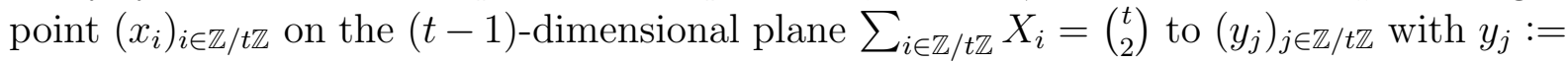
$\frac{1}{t}\left(x_{s j+k}-\left[x_{s(j+1)+k}-s\right]\right)$. Then $\phi$ maps into the $(t-1)$-dimensional plane $\sum_{j \in \mathbb{Z} / t \mathbb{Z}} Y_{j}=s$. Since $s, t$ are coprime, it is easy to check that $\phi:\left\{\sum_{i \in \mathbb{Z} / t \mathbb{Z}} X_{i}=\left(\begin{array}{l}t \\ 2\end{array}\right)\right\} \rightarrow\left\{\sum_{j \in \mathbb{Z} / t \mathbb{Z}} Y_{j}=s\right\}$ is injective, hence bijective.

Perhaps the most natural description of the inverse $\phi^{-1}$ is given (for $\ell \in \mathbb{Z} / t \mathbb{Z}$, noting that $\ell \mapsto s \ell+k$ is surjective modulo $t)$ by evaluating $\sum_{j=0}^{t-1}\left(\frac{t-1}{2}-j\right) y_{j+\ell} \cdot t$, i.e. the sum $\sum_{j=0}^{t-1}\left(\frac{t-1}{2}-j\right) s+\left(\frac{t-1}{2}-j\right) x_{s j+s \ell+k}-\left(\frac{t-1}{2}-j\right) x_{s(j+1)+s \ell+k}$, which telescopes to

$$
\frac{t-1}{2} x_{s \cdot 0+s \ell+k}+\frac{t-1}{2} x_{s \cdot t+s \ell+k}-\sum_{j=0}^{t-2} x_{s(j+1)+s \ell+k}=t x_{s \ell+k}-\left(\begin{array}{l}
t \\
2
\end{array}\right) .
$$

This yields the equality $\sum_{j=0}^{t-1}\left(\frac{t-1}{2}-j\right) y_{j+\ell}=x_{s \ell+k}-\frac{t-1}{2}$.

Having analyzed the ambient affine space, we now wish to show that $\phi$ restricts to a set bijection $A_{t}(s) \rightarrow \mathrm{TD}_{t}(s)$ for $\mathcal{C}_{s} \cap \mathcal{C}_{t}$, as well as the analogous bijection for $\mathcal{C}_{t}$. Suppose $\left(x_{i}\right) \in\left\{\sum_{i \in \mathbb{Z} / t \mathbb{Z}} X_{i}=\left(\begin{array}{c}t \\ 2\end{array}\right)\right\}$ corresponds under $\phi$ to $\left(y_{j}\right) \in\left\{\sum_{j \in \mathbb{Z} / t \mathbb{Z}} Y_{j}=s\right\}$; then we make the following observations.

1. Since $s, t$ are coprime, the inequalities $x_{i} \geqslant x_{i+s}-s$ hold for all $i \in \mathbb{Z} / t \mathbb{Z}$ if and only if $y_{j} \geqslant 0$ for all $j \in \mathbb{Z} / t \mathbb{Z}$;

2. The identity $\sum_{j=0}^{t-1} j y_{j+\ell}=k-x_{k+s \ell}$ follows (for any $\ell \in \mathbb{Z} / t \mathbb{Z}$ ) from the telescoping sum above (substituting $\sum_{j=0}^{t-1} y_{j+\ell}=s$ and $k=\frac{t-1}{2}+\frac{t-1}{2} \cdot s$ );

3. If $x_{i} \equiv i(\bmod t)$ for all $i \in \mathbb{Z} / t \mathbb{Z}$, then $\sum j y_{j}=k-x_{k} \equiv 0(\bmod t)$ and $y_{j} \equiv 0$ $(\bmod 1)$ for all $j \in \mathbb{Z} / t \mathbb{Z}$;

4. Suppose $\sum_{j=0}^{t-1} j y_{j}\left(=k-x_{k}\right)$ is $0(\bmod t)$, i.e. $x_{k} \equiv k(\bmod t)$, and further $y_{j} \equiv 0$ $(\bmod 1)$ for all $j \in \mathbb{Z} / t \mathbb{Z}$. Then for any $\ell \in \mathbb{Z}$, we have

$$
x_{k+s \ell}=k-\sum_{j=0}^{t-1} j y_{j+\ell}=k+s \ell-\sum_{j=0}^{t-1}(j+\ell) y_{j+\ell} \equiv k+s \ell-\sum_{j \in \mathbb{Z} / t \mathbb{Z}} j y_{j} \equiv k+s \ell \quad(\bmod t),
$$

so $x_{i} \equiv i(\bmod t)$ for all $i \in \mathbb{Z} / t \mathbb{Z}$ (as $s, t$ are coprime). (Alternatively, we could look at the partial sums $z_{0}+\cdots+z_{\ell-1}=\frac{1}{t}\left(x_{k}-x_{k+\ell s}+\ell s\right)$.) 
The first and third items show that $\phi$ maps $A_{t}(s)$ into $\operatorname{TD}_{t}(s)$. The first and fourth items show that $\phi^{-1}$ maps $\operatorname{TD}_{t}(s)$ into $A_{t}(s)$. (We are using the fact that $\phi$ bijects a superset of $A_{t}(s)$ to a superset of $\mathrm{TD}_{t}(s)$.) So $\phi$ bijects $A_{t}(s)$ to $\mathrm{TD}_{t}(s)$, establishing the change of coordinates for $\mathcal{C}_{s} \cap \mathcal{C}_{t}$. Similarly, considering only the third and fourth items (ignoring the first item) establishes the change of coordinates for $\mathcal{C}_{t}$.

Finally, the explicit formula for the inverse $\phi^{-1}$ establishes the desired description of the inverse $\left(a_{t, i}\right)_{i \in \mathbb{Z} / t \mathbb{Z}}=\phi^{-1}\left(z_{t, j}\right)_{j \in \mathbb{Z} / t \mathbb{Z}}$ of the isomorphism.

Remark 4.5. Already in this proof we have seen the 'cyclic shifts identity' $\sum_{j=0}^{t-1} j y_{j+\ell} \equiv$ $-s \ell+\sum_{j \in \mathbb{Z} / t \mathbb{Z}} j y_{j}(\bmod t)$ for $t$ integers $\left(y_{j}\right)_{j \in \mathbb{Z} / t \mathbb{Z}}$ summing to $s$, which will feature more prominently in Proposition 4.7.

Corollary 4.6 (Weak version of Anderson's theorem [5]). $A_{t}(s)$ and $\mathrm{TD}_{t}(s)$ are discrete bounded sets, hence finite. In particular, there are finitely many $(s, t)$-cores, so the sums in Theorems 1.1, 1.2, 1.3, 1.4 are finite and well-defined.

\subsection{Cyclic shifts in the $z$-coordinates}

Johnson [21] relies on cyclic symmetry in his proofs of Anderson's theorem [5] (that there are exactly $\frac{1}{s+t}\left(\begin{array}{c}s+t \\ s\end{array}\right)$ distinct simultaneous $(s, t)$-cores) and Armstrong's general conjecture (Theorem 1.1). For Theorems 1.1 and 1.3, we also rely on the following 'cyclic shifts' argument.

Proposition 4.7 (c.f. [21, proofs of Corollary 3.6 and Theorem 3.7]). Fix coprime $s, t \geqslant$ 1. Let $f\left(X_{0}, \ldots, X_{t-1}\right)$ be a cyclic complex-valued function (i.e. for all $i \in \mathbb{Z} / t \mathbb{Z}$ we have $f\left(X_{0}, \ldots, X_{t-1}\right)=f\left(X_{i}, \ldots, X_{i+t-1}\right)$, with indices taken modulo $\left.t\right)$. Then

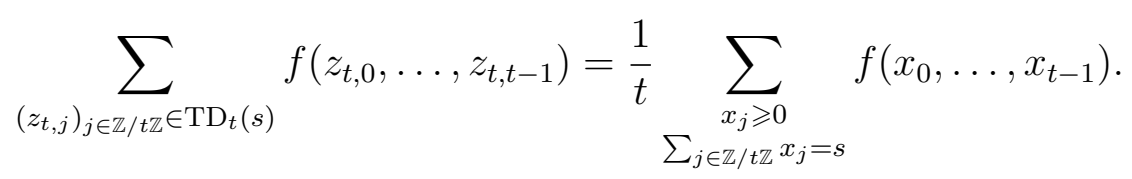

Proof. For any $t$ nonnegative integers $x_{0}, \ldots, x_{t-1} \geqslant 0$ (indexed modulo $t$ ) summing to $s$, the cyclic permutations $\left(x_{r}, \ldots, x_{r+t-1}\right)$ leave distinct residues via the 'cyclic shifts identity'

$$
\sum_{j \in \mathbb{Z} / t \mathbb{Z}} j x_{r+j} \equiv-r s+\sum_{j \in \mathbb{Z} / t \mathbb{Z}}(r+j) x_{r+j} \equiv-r s+\sum_{j \in \mathbb{Z} / t \mathbb{Z}} j x_{j} \quad(\bmod t),
$$

since $s$ is coprime to $t$. Thus each orbit of the cyclic $\mathbb{Z} / t \mathbb{Z}$-action contains exactly one point of $\operatorname{TD}_{t}(s)$, and since $f$ is cyclic (and the sums are over the finite sets $\operatorname{TD}_{t}(s)$ and $\left\{\left(x_{j}\right)_{j \in \mathbb{Z} / t \mathbb{Z}} \in \mathbb{Z}_{\geqslant 0}^{t}: \sum_{j \in \mathbb{Z} / t \mathbb{Z}} x_{j}=s\right\}$, by Corollary 4.6$)$, the result follows.

Corollary 4.8 (Relevant cyclic sums). Fix coprime $s, t \geqslant 1$. Then we evaluate the sum $\sum_{\left(z_{t, j}\right) \in \mathrm{TD}_{t}(s)} f\left(z_{t, 0}, \ldots, z_{t, t-1}\right)$ in the cases listed below, where the most important terms have been boxed. For a vector or weak composition $\mathbf{x}=\left(x_{i}\right)_{i \in \mathbb{Z} / t \mathbb{Z}}$ with $t$ components, define the sum $|\mathbf{x}|:=\sum_{i \in \mathbb{Z} / t \mathbb{Z}} x_{i}$ and (if appropriate) the multinomial coefficient $\left(\begin{array}{c}|\mathbf{x}| \\ \mathbf{x}\end{array}\right):=\left(\begin{array}{c}|\mathbf{x}| \\ x_{1}, \ldots, x_{t}\end{array}\right)$.

First we look at "exponential" cases. 
- $\frac{1}{t} \cdot t^{s}$ when $f=\left(\begin{array}{c}|\mathbf{x}| \\ \mathbf{x}\end{array}\right) \cdot 1$ (constant);

- $\frac{1}{t} \cdot s t^{s-1}$ when $f=\left(\begin{array}{c}|\mathbf{x}| \\ \mathbf{x}\end{array}\right) \cdot \frac{1}{t} \sum_{i \in \mathbb{Z} / t \mathbb{Z}} x_{i}$ (linear);

- $\frac{1}{t} \cdot s(s-1) t^{s-2}$ when $f=\left(\begin{array}{c}|\mathbf{x}| \\ \mathbf{x}\end{array}\right) \cdot \frac{1}{t} \sum_{i \in \mathbb{Z} / t \mathbb{Z}} x_{i}\left(x_{i}-1\right)$ or $f=\left(\begin{array}{c}|\mathbf{x}| \\ \mathbf{x}\end{array}\right) \cdot \frac{1}{t} \sum_{i \in \mathbb{Z} / t \mathbb{Z}} x_{i} x_{i+r}$ for some $r \not \equiv 0(\bmod t)$ (quadratic).

Next we look at "ordinary" cases.

- $\frac{1}{t} \cdot\left(\begin{array}{c}s+t-1 \\ t-1\end{array}\right)$ when $f=1$ (constant);

- $\frac{1}{t} \cdot\left(\begin{array}{c}s+t-1 \\ t\end{array}\right)$ when $f=\frac{1}{t} \sum_{i \in \mathbb{Z} / t \mathbb{Z}} x_{i}$ (linear);

- $\frac{1}{t} \cdot 2\left(\begin{array}{c}s+t-1 \\ t+1\end{array}\right)$ when $f=\frac{1}{t} \sum_{i \in \mathbb{Z} / t \mathbb{Z}} x_{i}\left(x_{i}-1\right)$ (square quadratic);

- $\frac{1}{t} \cdot\left(\begin{array}{c}s+t-1 \\ t+1\end{array}\right)$ when $f=\frac{1}{t} \sum_{i \in \mathbb{Z} / t \mathbb{Z}} x_{i} x_{i+r}$ for some $r \not \equiv 0(\bmod t)$ (mixed quadratic).

Remark 4.9. We will use these explicit (generating function) calculations below in the proofs of Theorems 1.1 and 1.3, instead of the coarser Ehrhart and Euler-Maclaurin theory language of Johnson [21]. (See Remarks 8.3 and 8.6 for further conceptual discussion.)

Proof of "exponential" cases. First we use Proposition 4.7 to reduce the cyclic sums in question over $\operatorname{TD}_{t}(s)$ to sums over the easier domain of $\left\{x_{i} \geqslant 0:|\mathbf{x}|=s\right\}$. On this easier domain, the standard tool of exponential generating functions $\left(\prod_{i \in \mathbb{Z} / t \mathbb{Z}} \exp \left(Z_{i} T\right)\right)$ suffices. Equivalently, one may directly differentiate the multinomial $\sum_{|\mathbf{x}|=s}\left(\begin{array}{c}|\mathbf{x}| \\ \mathbf{x}\end{array}\right) Z_{0}^{x_{0}} \cdots Z_{t-1}^{x_{t-1}}=$ $\left(Z_{0}+\cdots+Z_{t-1}\right)^{s}$. For example, by differentiating zero times we get $\sum_{|\mathbf{x}|=s}\left(\begin{array}{c}|\mathbf{x}| \\ \mathbf{x}\end{array}\right)=t^{s}$; by differentiating once we get $\sum_{|\mathbf{x}|=s}\left(\begin{array}{c}|\mathbf{x}| \\ \mathbf{x}\end{array}\right) x_{0}=s t^{s-1}$; and by differentiating twice we get $\sum_{|\mathbf{x}|=s}\left(\begin{array}{c}|\mathbf{x}| \\ \mathbf{x}\end{array}\right) x_{0}\left(x_{0}-1\right)=\sum_{|\mathbf{x}|=s}\left(\begin{array}{c}|\mathbf{x}| \\ \mathbf{x}\end{array}\right) x_{0} x_{r}=s(s-1) t^{s-2}$ (if $\left.t \nmid r\right)$.

Proof of "ordinary" cases. Once again we use Proposition 4.7 to reduce the cyclic sums in question over $\operatorname{TD}_{t}(s)$ to sums over the easier domain of $\left\{x_{i} \geqslant 0:|\mathbf{x}|=s\right\}$, where the standard tool of ordinary generating functions $\left(\prod_{i \in \mathbb{Z} / t \mathbb{Z}}\left(1-Z_{i} T\right)^{-1}\right)$ suffices. For example, by differentiating zero times we get $\sum_{|\mathbf{x}|=s} 1=\left[T^{s}\right](1-T)^{-t}=\left(\begin{array}{c}s+t-1 \\ t-1\end{array}\right)$; by differentiating once (with respect to $Z_{0}$ ) we get $\sum_{|\mathbf{x}|=s} x_{0}=\left[T^{s}\right] T(1-T)^{-t-1}=\left(\begin{array}{c}(s-1)+(t+1)-1 \\ (t+1)-1\end{array}\right)$; by differentiating twice with respect to $Z_{0}$ we get $\sum_{|\mathbf{x}|=s} x_{0}\left(x_{0}-1\right)=\left[T^{s}\right] 2 T^{2}(1-T)^{-t-2}=2\left(\begin{array}{c}s+t-1 \\ t+1\end{array}\right)$; or by differentiating once with respect to each of $Z_{0}, Z_{r}$ (if $t \nmid r$ ) we get $\sum_{|\mathbf{x}|=s} x_{0} x_{r}=$ $\left[T^{s}\right] T^{2}(1-T)^{-t-2}=\left(\begin{array}{c}s+t-1 \\ t+1\end{array}\right)$. 


\subsection{Size of a $t$-core}

The $c$ - and $a$ - coordinate versions of the following lemma could have been placed earlier, but we wish to use the $z$-coordinates, most relevant right before Section 5 . The $c$-coordinate version has been used in [19] and [12] to deduce certain properties of the generating function for $t$-cores.

Lemma 4.10 (c.f. [21, proof of Theorem 2.10]). Fix $t \geqslant 1$ and a $t$-core $\lambda$.

- In Johnson's $c_{t}$-coordinates, $|\lambda|=\sum_{i=0}^{t-1}\left(\frac{t}{2} c_{t, i}^{2}-\left(\frac{t-1}{2}-i\right) c_{t, i}\right)$, from [21, Theorem 2.10]; [19, Bijection 2]; [12], up to the linear relation $\sum_{i \in \mathbb{Z} / t \mathbb{Z}} c_{t, i}=0$.

- In Fayers' $a_{t}$-coordinates, $|\lambda|=-\frac{1}{24}\left(t^{2}-1\right)+\frac{1}{2 t} \sum_{i \in \mathbb{Z} / t \mathbb{Z}}\left[a_{t, i}-\frac{t-1}{2}\right]^{2}$ (a symmetric polynomial);

- Fix $s \geqslant 1$ coprime to $t$. In the extended $z_{t}$-coordinates,

$$
|\lambda|=-\frac{1}{24}\left(t^{2}-1\right)+\frac{1}{24}\left(t^{2}-1\right) \sum_{\ell \in \mathbb{Z} / t \mathbb{Z}} z_{t, \ell}^{2}+M_{2}\left(z_{t, 0}, \ldots, z_{t, t-1}\right)
$$

(a cyclic polynomial), where $M_{2} \in \mathbb{Z}\left[X_{0}, \ldots, X_{t-1}\right]$ is the "leftover" cyclic homogeneous quadratic with only 'mixed' terms (i.e. no square terms $X_{0}^{2}, \ldots, X_{t-1}^{2}$ ), and with coefficient sum $-\frac{1}{24} t\left(t^{2}-1\right)$.

In particular, in z-coordinates, the sum of the coefficients of $|\lambda|+\frac{1}{24}\left(t^{2}-1\right)$-the nonconstant (i.e. homogeneous quadratic) part of the z-expression-is 0 .

Remark 4.11. Our proofs of Theorems 1.1 and 1.3 rely crucially on the cyclic symmetry of the formula for the size $|\lambda|$ in the $z$-coordinates. (Johnson's proof of Theorem 1.1 does as well [21].) Due to the cyclic nature of the change of variables relating the $z$ - and $a$ coordinates (see Proposition 4.2), it suffices to understand why $|\lambda|$ is symmetric in the $a$-coordinates.

The following proof directly explains the (complete) symmetry in the $a$-coordinates, but one could also convert from the c-coordinates using Section 2.4.

Discrete calculus proof for a-coordinates. Split the partition into two halves by cutting along the $y$-axis (see Figure 3). We calculate the size of the partition (or area of the partition diagram) as

$$
|\lambda|=\sum_{\substack{x \in B^{\lambda} \\ x+\frac{1}{2}>0}}\left(x+\frac{1}{2}\right)-\sum_{\substack{x \notin B^{\lambda} \\ x+\frac{1}{2}<0}}\left(x+\frac{1}{2}\right) .
$$

As written this holds for any partition, but we want to simplify it further for $t$-cores $\lambda$. As we are working with the $a_{t, i}$-coordinates, we will break up the contributions by residue class modulo $t$. Fix $0 \leqslant i \leqslant t-1$, and define the $i+t \mathbb{Z}$ area contribution $F\left(a_{t, i}\right)$ (i.e. contribution in the sums above from $x$ congruent to $i(\bmod t))$; we will study how it changes as $a_{t, i}$ varies along the residue class $i+t \mathbb{Z}$. We have 


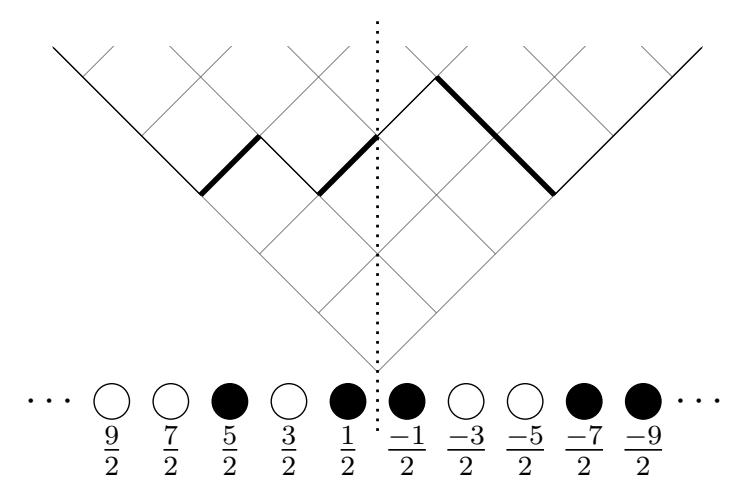

Figure 3: Russian notation for $\lambda=(3,2,2,0, \ldots)$, based on Figure 2. The $y$-axis (dotted) is distinguished for area computation: $|\lambda|=\frac{5}{2}+\frac{1}{2}-\left(\frac{-3}{2}\right)-\left(\frac{-5}{2}\right)$.

- $F(i)=0$ (no contribution when $\left.B^{\lambda} \cap(i+t \mathbb{Z})=\{\ldots, i-3 s, i-2 s, i-s\}\right)$;

- $F\left(a_{t, i}\right)-F\left(a_{t, i}-t\right)=\left[a_{t, i}-t\right]+\frac{1}{2}$ (note that this holds both in the 'positive/left case' $a_{t, i} \geqslant i+t$ and the 'negative/right case' $\left.a_{t, i} \leqslant i\right)$.

Standard discrete calculus yields $F\left(a_{t, i}\right)=G\left(a_{t, i}\right)-G(i)$, where $G(x)=\frac{1}{2 t} x(x-t)+\frac{1}{2} \cdot \frac{1}{t} x$. Completing the square gives $F\left(a_{t, i}\right)=\frac{1}{2 t}\left[a_{t, i}-\frac{t-1}{2}\right]^{2}-\frac{1}{2 t}\left[i-\frac{t-1}{2}\right]^{2}$.

To finish, we sum over all $0 \leqslant i \leqslant t-1$, using the identity $\frac{1}{2 t} \sum_{i=0}^{t-1}\left(i-\frac{t-1}{2}\right)^{2}=$ $\frac{1}{24}\left(t^{2}-1\right)$.

Proof of a-to- $z$ translation. Write $|\lambda|=-\frac{1}{24}\left(t^{2}-1\right)+\frac{1}{2 t} \cdot P$ for convenience. Proposition 4.2 (which holds for $t$-cores, not just $(s, t)$-cores) gives $a_{t, k+\ell s}-\frac{t-1}{2}=\sum_{j=0}^{t-1}\left(\frac{t-1}{2}-j\right) z_{t, j+\ell}$ for all $\ell \in \mathbb{Z} / t \mathbb{Z}$, since $s, t$ are coprime. Then

$$
P:=\sum_{i=0}^{t-1}\left[a_{t, i}-\frac{t-1}{2}\right]^{2}=\sum_{\ell \in \mathbb{Z} / t \mathbb{Z}}\left[a_{t, k+\ell s}-\frac{t-1}{2}\right]^{2}=\sum_{\ell \in \mathbb{Z} / t \mathbb{Z}}\left[\sum_{j=0}^{t-1}\left(\frac{t-1}{2}-j\right) z_{t, j+\ell}\right]^{2}
$$

is a cyclic quadratic polynomial in the $z_{t, \ell}$, so $P=C(t) \sum_{\ell \in \mathbb{Z} / t \mathbb{Z}} z_{t, \ell}^{2}+H\left(z_{t, 0}, \ldots, z_{t, t-1}\right)$ for some constant $C$ depending only on $t$, and a cyclic homogeneous quadratic $H \in$ $\mathbb{Z}\left[X_{0}, \ldots, X_{t-1}\right]$ with no 'square terms' (i.e. $X_{0}^{2}, \ldots, X_{t-1}^{2}$ ). We make the following calculations.

- $P$ vanishes when $a_{t, \ell}=\frac{t-1}{2}$ for all $\ell \in \mathbb{Z} / t \mathbb{Z}$, or equivalently when the $z$-coordinates are all equal. So the sum of $z$-coefficients of $|\lambda|+\frac{1}{24}\left(t^{2}-1\right)=\frac{1}{2 t} \cdot P$ is 0 .

- $C(t)=\sum_{j=0}^{t-1}\left(\frac{t-1}{2}-j\right)^{2}=\frac{1}{12} t\left(t^{2}-1\right)$.

- $H(1, \ldots, 1)=P(1, \ldots, 1)-C(t) \sum_{\ell \in \mathbb{Z} / t \mathbb{Z}} 1^{2}=0-t C(t)=-\frac{1}{12} t^{2}\left(t^{2}-1\right)$.

Substituting $P$ into $|\lambda|=-\frac{1}{24}\left(t^{2}-1\right)+\frac{1}{2 t} \cdot P$ finishes the job. 


\section{$5 \quad$ Proofs of general conjectures}

In this section, we first give a proof of Armstrong's general conjecture by direct computation. We then use the same methods to prove Fayers' general conjecture.

Proof of Theorem 1.1 by direct computation. We use z-coordinates. By Corollary 4.8, the denominator is just $\sum_{\mathrm{TD}_{t}(s)} 1=\frac{1}{t}\left(\begin{array}{c}s+t-1 \\ t-1\end{array}\right)$ (the number of $(s, t)$-cores).

Using Lemma 4.10 and Corollary 4.8 with the identity $x^{2}=x(x-1)+x$, the numerator $\sum_{\mathrm{TD}_{t}(s)}|\lambda|=\sum_{\mathrm{TD}_{t}(s)}\left(-\frac{1}{24}\left(t^{2}-1\right) \cdot 1+\frac{1}{24}\left(t^{2}-1\right) \cdot \sum_{\ell \in \mathbb{Z} / t \mathbb{Z}} z_{t, \ell}^{2}+M_{2}\left(z_{t, \ell}\right)\right)$ becomes

$$
\begin{aligned}
& -\frac{t^{2}-1}{24} \cdot \frac{1}{t}\left(\begin{array}{c}
N \\
t-1
\end{array}\right)+\frac{t^{2}-1}{24} \cdot t \cdot \frac{1}{t}\left[\left(\begin{array}{c}
N \\
t
\end{array}\right)+2\left(\begin{array}{c}
N \\
t+1
\end{array}\right)\right]-\frac{t\left(t^{2}-1\right)}{24} \cdot \frac{1}{t}\left(\begin{array}{c}
N \\
t+1
\end{array}\right) \\
& =\frac{t^{2}-1}{24} \cdot \frac{1}{t}\left(\begin{array}{c}
N \\
t-1
\end{array}\right)\left[-1+t \cdot \frac{s}{t}+t \cdot \frac{s(s-1)}{t(t+1)}\right]
\end{aligned}
$$

where we have suppressed $N:=s+t-1$ and used $\left(\begin{array}{c}N \\ t\end{array}\right)=\frac{s}{t}\left(\begin{array}{c}N \\ t-1\end{array}\right)$ and $\left(\begin{array}{c}N \\ t+1\end{array}\right)=\frac{s-1}{t+1}\left(\begin{array}{c}N \\ t\end{array}\right)$ (viewed as polynomial identities in $s$, for fixed $t \geqslant 1$ ). Finally, dividing the numerator expression by the denominator $\frac{1}{t}\left(\begin{array}{c}N \\ t-1\end{array}\right)$ yields $\frac{1}{24}\left(t^{2}-1\right)\left(-1+s+\frac{s(s-1)}{t+1}\right)$, which simplifies to $\frac{1}{24}(t-1)(t+1) \frac{s-1}{t+1}[(t+1)+s]=\frac{1}{24}(s-1)(t-1)(s+t+1)$.

The same technique proves Fayers' general conjecture.

Proof of Theorem 1.3. In $z$-coordinates, the stabilizer $\operatorname{Stab}_{G_{s, t}}(\lambda)$ has size $\prod_{i \in \mathbb{Z} / t \mathbb{Z}} z_{t, i}$ ! (by Corollary 4.4). By Corollary 4.8, the denominator times $s$ ! is just $\sum_{\mathrm{TD}_{t}(s)}\left(\begin{array}{c}\left|\mathbf{z}_{\mathbf{t}}\right| \\ \mathbf{z}_{\mathbf{t}}\end{array}\right)=\frac{1}{t} \cdot t^{s}=$ $t^{s-1}$.

Using Lemma 4.10 and Corollary 4.8 with the identity $x^{2}=x(x-1)+x$, the numerator times s!, i.e. $\sum_{\mathrm{TD}_{t}(s)}\left(\begin{array}{c}\left|\mathbf{z}_{\mathbf{t}}\right| \\ \mathbf{z}_{\mathbf{t}}\end{array}\right)|\lambda|=\sum_{\mathrm{TD}_{t}(s)}\left(\begin{array}{c}\left|\mathbf{z}_{\mathbf{t}}\right| \\ \mathbf{z}_{\mathbf{t}}\end{array}\right)\left(-\frac{1}{24}\left(t^{2}-1\right) \cdot 1+\frac{1}{24}\left(t^{2}-1\right) \cdot \sum_{\ell \in \mathbb{Z} / t \mathbb{Z}} z_{t, \ell}^{2}+\right.$ $\left.M_{2}\left(z_{t, \ell}\right)\right)$, becomes

$$
-\frac{1}{24}\left(t^{2}-1\right) \cdot \frac{1}{t} \cdot t^{s}+\frac{1}{24}\left(t^{2}-1\right) \cdot\left[s t^{s-1}+s(s-1) t^{s-2}\right]-\frac{1}{24}\left(t^{2}-1\right) \cdot s(s-1) t^{s-2},
$$

which simplifies to $\frac{1}{24}\left(t^{2}-1\right) t^{s-1}(s-1)$. Finally, dividing ( $s$ ! times the) numerator by $(s$ ! times the) denominator yields the desired ratio of $\frac{1}{24}(s-1)\left(t^{2}-1\right)$.

Remark 5.1. Conceptually, the $s(s-1) t^{s-2}$ coefficients cancel because they collect to form the sum of the coefficients of $|\lambda|+\frac{1}{24}\left(t^{2}-1\right)$, which is 0 as noted in Lemma 4.10.

\section{Self-conjugate analogs}

Using almost the same methods as before, we build up to proofs of Theorems 1.2 and 1.4 in Section 6.4. However, we no longer need a cyclic shifts argument, because the parameterization of self-conjugate cores (see Proposition 6.11) is simpler. 


\subsection{Background: beta-sets, charges, $s$-sets, and conjugation}

Proposition 6.1 (c.f. $\left[15\right.$, Lemma 4.3]). Fix $x \in \mathbb{Z}$. Then $x \in B^{\bar{\lambda}}$ if and only if $-1-x \notin B^{\lambda}$.

Proposition 6.2 (Extension of Fayers [15, Lemma 4.6]). Fix $s \geqslant 1$ and $i \in \mathbb{Z} / s \mathbb{Z}$. Then $c_{s, i}(\bar{\lambda})=-c_{s,-1-i}(\lambda)$ holds for all partitions $\lambda$.

Proof. Substituting Proposition 6.1 into Definition 2.4 for $c_{s, i}(\bar{\lambda})$ gives

$c_{s, i}(\bar{\lambda})=\#\left[(-1-i+s \mathbb{Z}) \cap\left(-1-B^{\lambda}\right) \cap\left\{x+\frac{1}{2}<0\right\}\right]-\#\left[(-1-i+s \mathbb{Z}) \cap\left(\mathbb{Z} \backslash-1-B^{\lambda}\right) \cap\left\{x+\frac{1}{2}>0\right\}\right]$.

Applying the involution $x \mapsto-1-x$ recovers the definition of $-c_{s,-1-i}(\lambda)$.

But the $s$-core operation preserves $s$-charge (see Proposition 2.10), so Proposition 6.2 yields $c_{s, i}\left(\bar{\lambda}^{s}\right)=c_{s, i}(\bar{\lambda})=c_{s,-1-i}(\lambda)=c_{s,-1-i}\left(\lambda^{s}\right)=c_{s, i}\left(\overline{\lambda^{s}}\right)$. Since $c$-coordinates uniquely parameterize $s$-cores (Proposition 2.7), we obtain the following result.

Corollary 6.3. The s-core operation commutes with conjugation, i.e. $\bar{\lambda}^{s}=\overline{\lambda^{s}}$ for any partition $\lambda$.

The $c$-to- $a$ translation of Section 2.4 gives another corollary of Proposition 6.2.

Corollary 6.4 ([15, proof of Lemma 4.6]). Fix $\lambda \in \mathcal{C}_{s}$. Then $\bar{\lambda} \in \mathcal{C}_{s}$ as well, and $a_{s, i}(\bar{\lambda})=s-1-a_{s,-1-i}(\lambda)$ for all $i \in \mathbb{Z} / s \mathbb{Z}$, or equivalently $\mathcal{S}_{s}(\bar{\lambda})=s-1-\mathcal{S}_{s}(\lambda)$. In particular, $\lambda \in \mathcal{D}_{s}$ if and only if $\mathcal{S}_{s}(\lambda)=s-1-\mathcal{S}_{s}(\lambda)$, i.e. $\mathcal{S}_{s}$ is a symmetric $s$-set in Fayers' terminology.

\subsection{Background: $t$-cores of self-conjugate $s$-cores}

\subsubsection{Comparing $t$-cores of different self-conjugate $s$-cores}

The only necessary modification from the general case is the following self-conjugate version of Proposition 3.2.

Proposition 6.5 (Extension of Proposition 3.2). Fix any $s, t \geqslant 1$ and $\lambda \in \mathcal{D}_{s}$. Then $\lambda^{t} \in \mathcal{D}_{s} \cap \mathcal{D}_{t}$, and furthermore $\mathcal{S}_{s}\left(\lambda^{t}\right) \equiv \mathcal{S}_{s}(\lambda)$ ( $\left.\bmod t\right)$ (viewed as multisets of residues).

Direct alternative proof. Proposition 3.2 already shows everything except that $\lambda^{t}$ is selfconjugate. Strictly speaking, [15, Proposition 4.7] does prove this indirectly, but it is cleaner to use $\lambda=\bar{\lambda}$ and Corollary 6.3 to get $\lambda^{t}=\bar{\lambda}^{t}=\bar{\lambda}^{t}$.

\subsubsection{Self-conjugate level $t$ action}

We now present a self-conjugate analog of the group $G_{s, t}$ from Definition 3.7. 
Definition 6.6 (c.f. [15, Section 4.1]). Fix coprime $s, t \geqslant 1$. Let $H_{s, t}$ be the subgroup of $G_{s, t}$ consisting of $f \in G_{s, t}$ such that $f(-1-m)=-1-f(m)$ for all $m \in \mathbb{Z}$ (based on the involution $m \mapsto-1-m)$.

It is easy to check that $H_{s, t}$ has a group structure, and that it acts in the obvious way on the set of symmetric s-sets $\mathcal{S}_{s}(\lambda)$, or equivalently suitable beta-sets. This induces an action on $\mathcal{D}_{s}$, via Corollary 6.4.

Remark 6.7 (Different but equivalent definitions). We have not given Fayers' actual definition of the 'level $t$ action of the $s$-affine hyperoctahedral group', but rather one equivalent by [15, Propositions 4.1 and 4.2], and easier to work with for our purposes.

Remark 6.8 (Natural action?). It is not hard to show that $H_{s, t}$ is the subset of elements $f \in G_{s, t}$ that preserve $\mathcal{D}_{s}$.

We have following self-conjugate analog of Proposition 3.10, with analogous proof.

Proposition 6.9 (c.f. [15, proof of Proposition 4.9]). Let $\lambda, \mu$ be self-conjugate s-cores, and $\phi: \mathcal{S}_{s}(\lambda) \rightarrow \mathcal{S}_{s}(\mu)$ a bijection (of symmetric s-sets) that preserves residue classes modulo $t$, satisfying $\phi(m)+\phi(s-1-m)=s-1$ for all $m \in \mathcal{S}_{s}(\lambda)$. Then $\phi$ uniquely extends to an element $f \in H_{s, t}$ (under Definition 6.6).

We can now state a self-conjugate analog of Corollary 3.11 .

Corollary 6.10 ([15, Corollary 4.8]). Fix coprime $s, t \geqslant 1$. Then $\lambda, \mu \in \mathcal{D}_{s}$ lie in the same $H_{s, t}$-orbit if and only if $\lambda^{t}=\mu^{t}$. In other words, each $H_{s, t}$-orbit of $\mathcal{D}_{s}$ contains a unique $t$-core (hence self-conjugate $(s, t)$-core), and any $\lambda \in \mathcal{D}_{s}$ lies in $H_{s, t} \lambda^{t}$.

Sketch of more direct proof. Fix $\lambda, \mu \in \mathcal{D}_{s}$. Proposition 3.6 followed by Proposition 6.9 gives equivalence of $\lambda^{t}=\mu^{t}$ and $\mu \in H_{s, t} \lambda$. The re-phrasing follows by specializing to $\mu:=\lambda^{t}$ (where $\lambda^{t} \in \mathcal{D}_{s}$ follows from Proposition 6.5).

Definition 6.6 and Corollary 6.10 provide the background and context for Theorem 1.4 (stated in the introduction).

\subsection{Key inputs for computation}

In this section, we describe all the computational methods and results used to compute the sums in Theorems 1.2 and 1.4, roughly following the structure of Section 4.

\subsubsection{Johnson's $u$-coordinates versus Fayers' $t$-sets}

We will use the $z$-coordinates again (and the closely related $u$-coordinates) to get a simple expression (Proposition 6.12) for the $H_{s, t}$-stabilizers. Again, we not only review Johnson's $(s, t)$-core parameterization, but also give a modest extension to general $t$-cores.

Proposition 6.11 (c.f. [21, Section 3.3]: Johnson's $\boldsymbol{u}$-coordinates versus Fayers' $\boldsymbol{t}$-sets). Fix coprime $s, t \geqslant 1$. For convenience, let $s^{\prime}=\lfloor s / 2\rfloor$ and $t^{\prime}=\lfloor t / 2\rfloor$. The set $\mathcal{D}_{s} \cap \mathcal{D}_{t}$ of self-conjugate $(s, t)$-cores (viewed as self-conjugate $s$-cores within the set of $t$-cores) is parameterized by either of the sets $B_{t}(s), U_{t}(s)$, described as follows. 
- By Lemma 3.1, the set of $t$-sets $\mathcal{S}_{t}(\lambda)$ of self-conjugate $(s, t)$-cores $\lambda$, i.e. the subset $B_{t}(s)$ of points $\left(a_{t, i}\right)_{i \in \mathbb{Z} / t \mathbb{Z}} \in A_{t}(s)$ (specializing a-coordinates from Proposition 4.2) satisfying the additional symmetry $a_{t, i}+a_{t,-1-i}=t-1$ from Corollary 6.4 -this is [15, Lemma 4.6].

- The subset $U_{t}(s)$ of points $\left(z_{t, i}\right)_{i \in \mathbb{Z} / t \mathbb{Z}} \in \mathrm{TD}_{t}(s)$ (specializing z-coordinates from Proposition 4.2) satisfying the additional symmetry $z_{t, i}=z_{t,-i}$-this is [21, Lemma 3.9].

- In fact, as implicitly used in [21, proof of Lemma 3.10], the set $U_{t}(s)$ is canonically isomorphic to the set of lattice points $\left\{\left(u_{0}, u_{1}, \ldots, u_{t^{\prime}}\right) \in \mathbb{Z}_{\geqslant 0}^{1+t^{\prime}}: u_{0}+u_{1}+\cdots+u_{t^{\prime}}=\right.$ $\left.s^{\prime}\right\}$, with the isomorphism defined by $u_{0}:=\left\lfloor z_{t, 0} / 2\right\rfloor=s^{\prime}-\sum_{i=1}^{t^{\prime}} u_{i}$ and the following additional relations: $u_{i}:=z_{t, i}$ (for $1 \leqslant i \leqslant t^{\prime}$ ) if $t$ is odd; and $u_{t^{\prime}}:=z_{t, t^{\prime}} / 2$ and $u_{i}:=z_{t, i}\left(\right.$ for $\left.1 \leqslant i \leqslant t^{\prime}-1\right)$ if $t$ is even.

The isomorphism between these sets (also preserving the ambient linear and simplex structures) is the same as that described in Proposition 4.2.

Under the same affine change of coordinates, the set $\mathcal{D}_{t}$ of self-conjugate $t$-cores can be parameterized as follows.

- By Proposition 2.11, the set of t-sets $\mathcal{S}_{t}(\lambda)$ of self-conjugate $t$-cores $\lambda$, i.e. the subset of points $\left(a_{t, i}\right)_{i \in \mathbb{Z} / t \mathbb{Z}} \in \mathcal{C}_{t}$ satisfying the additional symmetry $a_{t, i}+a_{t,-1-i}=t-1$ from Corollary 6.4.

- In $z$-coordinates, the subset of points $\left(z_{t, i}\right)_{i \in \mathbb{Z} / t \mathbb{Z}} \in \mathcal{C}_{t}$ satisfying the additional symmetry $z_{t, i}=z_{t,-i}$.

- In u-coordinates, the set of lattice points $\left\{\left(u_{0}, u_{1}, \ldots, u_{t^{\prime}}\right) \in \mathbb{Z}^{1+t^{\prime}}: u_{0}+u_{1}+\cdots+u_{t^{\prime}}=\right.$ $\left.s^{\prime}\right\}$, with the isomorphism defined by $u_{0}:=\left\lfloor z_{t, 0} / 2\right\rfloor=s^{\prime}-\sum_{i=1}^{t^{\prime}} u_{i}$ and the following additional relations: $u_{i}:=z_{t, i}$ (for $1 \leqslant i \leqslant t^{\prime}$ ) if $t$ is odd; and $u_{t^{\prime}}:=z_{t, t^{\prime}} / 2$ and $u_{i}:=z_{t, i}$ (for $\left.1 \leqslant i \leqslant t^{\prime}-1\right)$ if $t$ is even.

Proof of a-to-z translation. As usual, let $k=\frac{1}{2}(s+1)(t-1)$. Define the map $\phi$ as in Proposition 4.2 , so for any $i \in \mathbb{Z} / t \mathbb{Z}$, the difference $t \cdot\left(y_{i}-y_{-i}\right)$ evaluates to

$\left(x_{s i+k}-x_{s i+s+k}+s\right)-\left(x_{-s i+k}-x_{-s i+s+k}+s\right)=\left(x_{s i+k}+x_{-s i+s+k}\right)-\left(x_{s i+s+k}+x_{-s i+k}\right)$.

Observe that $(s i+k)+(-s i+s+k)=(-s i+k)+(s i+s+k)=s+2 k \equiv-1(\bmod t)$ for all $i \in \mathbb{Z} / t \mathbb{Z}$. It follows (since $s$ is coprime to $t$ ) that $y_{i}=y_{-i}$ for all $i \in \mathbb{Z} / t \mathbb{Z}$ if and only if $x_{i}+x_{-1-i}$ is constant over $i \in \mathbb{Z} / t \mathbb{Z}$; if and only if $x_{i}+x_{-1-i}=t-1$ for all $i \in \mathbb{Z} / t \mathbb{Z}$ (because we are working in the plane $\sum_{i \in \mathbb{Z} / t \mathbb{Z}}\left(x_{i}+x_{-1-i}\right)=t(t-1)$ ).

Proof of $z$-to-u translation. If $y_{i}=y_{-i}$ for all $i \in \mathbb{Z} / t \mathbb{Z}$, then $0 y_{0}=0$ and $i y_{i}+(t-i) y_{t-i} \equiv$ $0(\bmod t)$ for all $i \in \mathbb{Z} / t \mathbb{Z}$.

If $t$ is odd, then we automatically get $\sum_{i \in \mathbb{Z} / t \mathbb{Z}} i y_{i} \equiv 0(\bmod t)$, clearly establishing $U_{t}(s)=\left\{\left(u_{0}, u_{1}, \ldots, u_{t^{\prime}}\right) \in \mathbb{Z}_{\geqslant 0}^{1+t^{\prime}}: u_{0}+u_{1}+\cdots+u_{t^{\prime}}=s^{\prime}\right\}$ under the canonical map given by $u_{i}:=z_{t, i}$ for $1 \leqslant i \leqslant t^{\prime}$ and $u_{0}:=\left\lfloor z_{t, 0} / 2\right\rfloor=s^{\prime}-\sum_{i=1}^{t^{\prime}} u_{i}\left(\right.$ from $\left.\sum_{i \in \mathbb{Z} / t \mathbb{Z}} z_{t, i}=s\right)$. 
If $t$ is even, then the vanishing of $\sum_{i \in \mathbb{Z} / t \mathbb{Z}} i y_{i} \equiv(t / 2) y_{t / 2}(\bmod t)$ modulo $t$ is equivalent to $y_{t^{\prime}} \equiv 0(\bmod 2)$. This establishes the isomorphism $U_{t}(s)=\left\{\left(u_{0}, u_{1}, \ldots, u_{t^{\prime}}\right) \in \mathbb{Z}_{\geqslant 0}^{1+t^{\prime}}\right.$ : $\left.u_{0}+u_{1}+\cdots+u_{t^{\prime}}=s^{\prime}\right\}$ under the canonical map given by $u_{t^{\prime}}=z_{t, t^{\prime}} / 2 ; u_{i}:=z_{t, i}$ for $1 \leqslant i \leqslant t^{\prime}-1$; and and $u_{0}:=\left\lfloor z_{t, 0} / 2\right\rfloor=s^{\prime}-\sum_{i=1}^{t^{\prime}} u_{i}\left(\right.$ from $\left.\sum_{i \in \mathbb{Z} / t \mathbb{Z}} z_{t, i}=s\right)$.

The previous two paragraphs prove the result for $\mathcal{D}_{s} \cap \mathcal{D}_{t}$. The same $z$-to- $u$ conversion works for $\mathcal{D}_{t}$ as well, except one ignores the inequality conditions $u_{i} \geqslant 0$ (which come from the conditions $z_{t, i} \geqslant 0$ of $\left.\operatorname{TD}_{t}(s)\right)$.

\subsubsection{Size of the stabilizer of a self-conjugate $s$-core}

The main new observation for Theorem 1.4 is the following computational simplification of Fayers' formula for the size of the stabilizer of a self-conjugate $s$-core, based on Proposition 3.4. This is the self-conjugate analog of Corollary 4.4 to Proposition 4.1.

Proposition 6.12 (c.f. Fayers [15, Proposition 4.9]). Fix coprime $s, t \geqslant 1$, and $\lambda \in \mathcal{D}_{s}$. For convenience, let $t^{\prime}=\lfloor t / 2\rfloor$.

1. If $t$ is odd, then $\left|\operatorname{Stab}_{H_{s, t}}(\lambda)\right|$ equals

$$
2^{\left\lfloor z_{t, 0}\left(\lambda^{t}\right) / 2\right\rfloor}\left\lfloor z_{t, 0}\left(\lambda^{t}\right) / 2\right\rfloor ! \prod_{i=1}^{t^{\prime}} z_{t, i}\left(\lambda^{t}\right) !=2^{u_{0}\left(\lambda^{t}\right)} \prod_{i=0}^{t^{\prime}} u_{i}\left(\lambda^{t}\right) ! .
$$

2. If $t$ is even (so $s$ is odd), then $\left|\operatorname{Stab}_{H_{s, t}}(\lambda)\right|$ equals

$$
2^{\left\lfloor z_{t, 0}\left(\lambda^{t}\right) / 2\right\rfloor}\left\lfloor z_{t, 0}\left(\lambda^{t}\right) / 2\right\rfloor ! 2^{\left\lfloor z_{t, t^{\prime}}\left(\lambda^{t}\right) / 2\right\rfloor}\left\lfloor z_{t, t^{\prime}}\left(\lambda^{t}\right) / 2\right\rfloor ! \prod_{i=1}^{t^{\prime}-1} z_{t, i}\left(\lambda^{t}\right) !=2^{u_{0}\left(\lambda^{t}\right)+u_{t^{\prime}}\left(\lambda^{t}\right)} \prod_{i=0}^{t^{\prime}} u_{i}\left(\lambda^{t}\right) !
$$

Proof. Fix $\lambda \in \mathcal{D}_{s}$. Fayers uses Proposition 6.9 to compute $\left|\operatorname{Stab}_{H_{s, t}}(\lambda)\right|$ as the number of permutations $\pi$ of the symmetric $s$-set $\mathcal{S}_{s}(\lambda)$ with $\pi(s-1-m)=s-1-\pi(m)$ and $\pi(m) \equiv m(\bmod t)$ for all $m \in \mathcal{S}_{s}(\lambda)$, i.e. the product of the following individual contributions:

- $\left|\mathcal{S}_{s}(\lambda) \cap(j+t \mathbb{Z})\right|$ ! = $\left|\mathcal{S}_{s}(\lambda) \cap(s-1-j+t \mathbb{Z})\right|$ ! for each pair of distinct residues $\{j, s-1-j\}(\bmod t)$;

- $2^{\left\lfloor\frac{1}{2}\left|\mathcal{S}_{s}(\lambda) \cap(j+t \mathbb{Z})\right|\right\rfloor}\left\lfloor\frac{1}{2}\left|\mathcal{S}_{s}(\lambda) \cap(j+t \mathbb{Z})\right|\right\rfloor$ ! for each residue $j(\bmod t)$ with $j \equiv s-1-j$ $(\bmod t)$.

Fayers evaluates the product in his own way, but it will be easier for us to directly translate to $z$-coordinates via

$$
z_{t, i}\left(\lambda^{t}\right)=\frac{1}{t}\left(a_{t, s i+k}\left(\lambda^{t}\right)-\left[a_{t, s(i+1)+k}\left(\lambda^{t}\right)-s\right]\right)=\left|\mathcal{S}_{s}(\lambda) \cap(s i+s+k+t \mathbb{Z})\right|
$$

from Corollary 4.4 (where $k=\frac{1}{2}(s+1)(t-1)$ as usual), and then to $u$-coordinates via Proposition 6.11 (as we have $\lambda^{t} \in \mathcal{D}_{s} \cap \mathcal{D}_{t}$ by Proposition 6.5). Observe that $2 s+2 k \equiv s-1$ $(\bmod t)$. 
1. If $t$ is odd, then the only residue $j$ with $j \equiv s-1-j(\bmod t)$ is $2^{-1}(s-1)$ $(\bmod t)$. It follows that the residues $s i+s+k+t \mathbb{Z}$, for $0 \leqslant i \leqslant \frac{t-1}{2}=t^{\prime}$, form a set of representatives of the pairs of residues $\{j, s-1-j\}(\bmod t)$, with $i=0$ corresponding to $j=s+k \equiv 2^{-1}(s-1)(\bmod t)$. Thus $\left|\operatorname{Stab}_{H_{s, t}}(\lambda)\right|$ equals the product $2^{\left\lfloor z_{t, 0}\left(\lambda^{t}\right) / 2\right\rfloor}\left\lfloor z_{t, 0}\left(\lambda^{t}\right) / 2\right\rfloor ! \prod_{i=1}^{t^{\prime}} z_{t, i}\left(\lambda^{t}\right) !$.

2. If $t$ is even (so $s$ is odd), then the residues $j$ with $j \equiv s-1-j(\bmod t)$ are $\frac{s-1}{2}+t \mathbb{Z}$ and $\frac{s-1}{2}+\frac{t}{2}+t \mathbb{Z}$. It follows that the residues $s i+s+k+t \mathbb{Z}$, for $0 \leqslant i \leqslant \frac{t}{2}=t^{\prime}$, form a set of representatives of the pairs of residues $\{j, s-1-j\}(\bmod t)$, with $i=0$ corresponding to $j=s+k=s+(t-1) \frac{s+1}{2} \equiv \frac{s-1}{2}(\bmod t)$ and $i=\frac{t}{2}=t^{\prime}$ corresponding to $j=s \frac{t}{2}+s+k \equiv 1 \frac{t}{2}+\frac{s-1}{2}=\frac{s-1}{2}+\frac{t}{2}(\bmod t)$. Thus $\left|\operatorname{Stab}_{H_{s, t}}(\lambda)\right|$ equals the product $2^{\left\lfloor z_{t, 0}\left(\lambda^{t}\right) / 2\right\rfloor}\left\lfloor z_{t, 0}\left(\lambda^{t}\right) / 2\right\rfloor ! 2^{\left\lfloor z_{t, t^{\prime}}\left(\lambda^{t}\right) / 2\right\rfloor}\left\lfloor z_{t, t^{\prime}}\left(\lambda^{t}\right) / 2\right\rfloor ! \prod_{i=1}^{t^{\prime}-1} z_{t, i}\left(\lambda^{t}\right) !$.

Finally, in the odd $t$ case, we can translate to $u$-coordinates using $u_{0}=\left\lfloor z_{t, 0} / 2\right\rfloor$ and $u_{i}=z_{t, i}$ for $1 \leqslant i \leqslant t^{\prime}$. In the even case, we instead use $z_{t, t^{\prime}}=2 u_{t^{\prime}}, u_{0}=\left\lfloor z_{t, 0} / 2\right\rfloor$, and $u_{i}=z_{t, i}$ for $1 \leqslant i \leqslant t^{\prime}-1$.

\subsubsection{Evaluating quadratic sums in $u$-coordinates}

We will evaluate several special $u$-coordinate sums in our proofs of Theorems 1.2 and 1.4 using the following self-conjugate analog of Corollary 4.8 .

Proposition 6.13. Fix coprime $s, t \geqslant 1$. Define $U_{t}(s)$ as in Proposition 6.11 (we will freely switch between $z$-coordinates and $u$-coordinates parameterizing self-conjugate $(s, t)$ cores as appropriate). For convenience, let $s^{\prime}=\lfloor s / 2\rfloor$ and $t^{\prime}=\lfloor t / 2\rfloor$, and let $[s]_{2}:=$ $s-2 s^{\prime} \in\{0,1\}$. Then we evaluate the sum $\sum_{\left(z_{t, j}\right) \in U_{t}(s)} f\left(z_{t, 0}, \ldots, z_{t, t-1}\right)$ in the cases listed below, where the most important terms have been boxed.

First we look at 'modified exponential' cases for odd $t$, freely using $z_{t, 0}=2 u_{0}+[s]_{2}$.

1. $\left(1 \cdot 2^{-1}+t^{\prime} \cdot 1\right)^{s^{\prime}}=\left(\frac{t}{2}\right)^{s^{\prime}}$ when ( $t$ is odd and) $f=2^{-u_{0}}\left(\begin{array}{c}|\mathbf{u}| \\ \mathbf{u}\end{array}\right) \cdot 1$, where $\mathbf{u}=\left(u_{0}, \ldots, u_{t^{\prime}}\right)$;

2. $s^{\prime}\left(\frac{t}{2}\right)^{s^{\prime}-1}$ when $f=2^{-u_{0}}\left(\begin{array}{c}|\mathbf{u}| \\ \mathbf{u}\end{array}\right) \cdot u_{i}$ for some $1 \leqslant i \leqslant t^{\prime}$;

3. $s^{\prime}\left(s^{\prime}-1\right)\left(\frac{t}{2}\right)^{s^{\prime}-2}$ when either $f=2^{-u_{0}}\left(\begin{array}{c}|\mathbf{u}| \\ \mathbf{u}\end{array}\right) \cdot u_{i}\left(u_{i}-1\right)$ for some $1 \leqslant i \leqslant t^{\prime}$ or $f=2^{-u_{0}}\left(\begin{array}{c}|\mathbf{u}| \\ \mathbf{u}\end{array}\right) \cdot u_{i} u_{j}$ for some distinct $1 \leqslant i<j \leqslant t^{\prime}$;

4. $[s]_{2}^{2}\left(\frac{t}{2}\right)^{s^{\prime}}+\left(2+2[s]_{2}\right) s^{\prime}\left(\frac{t}{2}\right)^{s^{\prime}-1}+s^{\prime}\left(s^{\prime}-1\right)\left(\frac{t}{2}\right)^{s^{\prime}-2}$ (comes from $[s]_{2}^{2}\left(\frac{t}{2}\right) s^{s^{\prime}}+\left(4+4[s]_{2}\right)$. $\left.2^{-1} s^{\prime}\left(\frac{t}{2}\right)^{s^{\prime}-1}+4 \cdot 2^{-2} s^{\prime}\left(s^{\prime}-1\right)\left(\frac{t}{2}\right)^{s^{\prime}-2}\right)$ when $f=2^{-u_{0}}\left(\begin{array}{c}|\mathbf{u}| \\ \mathbf{u}\end{array}\right) \cdot z_{t, 0}^{2}=2^{-u_{0}}\left(\begin{array}{c}|\mathbf{u}| \\ \mathbf{u}\end{array}\right) \cdot\left([s]_{2}^{2}+\right.$ $\left.\left(4+4[s]_{2}\right) u_{0}+4 u_{0}\left(u_{0}-1\right)\right)$ for some $1 \leqslant i \leqslant t^{\prime} ;$

5. $[s]_{2} s^{\prime}\left(\frac{t}{2}\right)^{s^{\prime}-1}+s^{\prime}\left(s^{\prime}-1\right)\left(\frac{t}{2}\right)^{s^{\prime}-2}$ (comes from $[s]_{2} s^{\prime}\left(\frac{t}{2}\right)^{s^{\prime}-1}+2 \cdot 2^{-1} s^{\prime}\left(s^{\prime}-1\right)\left(\frac{t}{2}\right)^{s^{\prime}-2}$ ) when $f=2^{-u_{0}}\left(\begin{array}{c}|\mathbf{u}| \\ \mathbf{u}\end{array}\right) \cdot z_{t, 0} u_{i}=2^{-u_{0}}\left(\begin{array}{c}|\mathbf{u}| \\ \mathbf{u}\end{array}\right) \cdot\left([s]_{2} u_{i}+2 u_{0} u_{i}\right)$ for some $1 \leqslant i \leqslant t^{\prime}$. 
Next we look at 'modified exponential' cases for even $t$, freely using $z_{t, 0}=2 u_{0}+[s]_{2}$ and $z_{t, t^{\prime}}=2 u_{t^{\prime}}$.

1. $\left(1 \cdot 2^{-1}+\left(\frac{t}{2}-1\right) \cdot 1+2^{-1}\right)^{s^{\prime}}=\left(\frac{t}{2}\right)^{s^{\prime}}$ when ( $t$ is even and) $f=2^{-u_{0}-u_{t^{\prime}}}\left(\begin{array}{c}|\mathbf{u}| \\ \mathbf{u}\end{array}\right) \cdot 1$;

2. $s^{\prime}\left(\frac{t}{2}\right)^{s^{\prime}-1}$ when $f=2^{-u_{0}-u_{t^{\prime}}}\left(\begin{array}{c}|\mathbf{u}| \\ \mathbf{u}\end{array}\right) \cdot \overline{u_{i}}$ for some $1 \leqslant i \leqslant t^{\prime}-1$;

3. $s^{\prime}\left(s^{\prime}-1\right)\left(\frac{t}{2}\right)^{s^{\prime}-2}$ when either $f=2^{-u_{0}-u_{t^{\prime}}}\left(\begin{array}{c}|\mathbf{u}| \\ \mathbf{u}\end{array}\right) \cdot u_{i}\left(u_{i}-1\right)$ for some $1 \leqslant i \leqslant t^{\prime}-1$ or $f=2^{-u_{0}-u_{t^{\prime}}}\left(\begin{array}{c}\mathbf{u} \mid \\ \mathbf{u}\end{array}\right) \cdot u_{i} u_{j}$ for some distinct $1 \leqslant i<j \leqslant t^{\prime}-1$;

4. $2 \cdot 2^{-1} s^{\prime}\left(s^{\prime}-1\right)\left(\frac{t}{2}\right)^{s^{\prime}-2}=s^{\prime}\left(s^{\prime}-1\right)\left(\frac{t}{2}\right)^{s^{\prime}-2}$ when $f=2^{-u_{0}-u_{t^{\prime}}}\left(\begin{array}{c}|\mathbf{u}| \\ \mathbf{u}\end{array}\right) \cdot z_{t, t^{\prime}} u_{i}=2$.

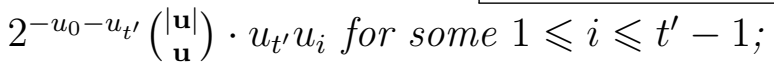

5. $2 \cdot 2^{-1} s^{\prime}\left(\frac{t}{2}\right)^{s^{\prime}-1}=s^{\prime}\left(\frac{t}{2}\right)^{s^{\prime}-1}$ when $f=2^{-u_{0}-u_{t^{\prime}}}\left(\begin{array}{c}|\mathbf{u}| \\ \mathbf{u}\end{array}\right) \cdot z_{t, t^{\prime}}=2 \cdot 2^{-u_{0}-u_{t^{\prime}}}\left(\begin{array}{c}|\mathbf{u}| \\ \mathbf{u}\end{array}\right) \cdot u_{t^{\prime}}$;

6. $2^{2} \cdot 2^{-2} s^{\prime}\left(s^{\prime}-1\right)\left(\frac{t}{2}\right) s^{s^{\prime}-2}+2^{2} \cdot 2^{-1} s^{\prime}\left(\frac{t}{2}\right)^{s^{\prime}-1}=s^{\prime}\left(s^{\prime}-1\right)\left(\frac{t}{2}\right)^{s^{\prime}-2}+2 s^{\prime}\left(\frac{t}{2}\right)^{s^{\prime}-1}$ when $f=$ $2^{-u_{0}-u_{t^{\prime}}\left(\begin{array}{c}|\mathbf{u}| \\ \mathbf{u}\end{array}\right) \cdot z_{t, t^{\prime}}^{2}}=2^{2} \cdot 2^{-u_{0}-u_{t^{\prime}}}\left(\begin{array}{c}|\mathbf{u}| \\ \mathbf{u}\end{array}\right)\left[u_{t^{\prime}}\left(u_{t^{\prime}}-1\right)+u_{t^{\prime}}\right]$;

7. $[s]_{2}^{2}\left(\frac{t}{2}\right)^{s^{\prime}}+\left(2+2[s]_{2}\right) s^{\prime}\left(\frac{t}{2}\right) s^{s^{\prime}-1}+s^{\prime}\left(s^{\prime}-1\right)\left(\frac{t}{2}\right)^{s^{\prime}-2}$ (comes from $[s]_{2}^{2}\left(\frac{t}{2}\right) s^{\prime}+\left(4+4[s]_{2}\right)$. $\left.2^{-1} s^{\prime}\left(\frac{t}{2}\right)^{s^{\prime}-1}+4 \cdot 2^{-2} s^{\prime}\left(s^{\prime}-1\right)\left(\frac{t}{2}\right)^{s^{\prime}-2}\right)$ when $f=2^{-u_{0}-u_{t^{\prime}}}\left(\begin{array}{c}|\mathbf{u}| \\ \mathbf{u}\end{array}\right) \cdot z_{t, 0}^{2}=2^{-u_{0}-u_{t^{\prime}}}\left(\begin{array}{c}|\mathbf{u}| \\ \mathbf{u}\end{array}\right) \cdot$ $\left([s]_{2}^{2}+\left(4+4[s]_{2}\right) u_{0}+4 u_{0}\left(u_{0}-1\right)\right)$ for some $1 \leqslant i \leqslant t^{\prime} ;$

8. $[s]_{2} s^{\prime}\left(\frac{t}{2}\right)^{s^{\prime}-1}+s^{\prime}\left(s^{\prime}-1\right)\left(\frac{t}{2}\right)^{s^{\prime}-2}$ (comes from $[s]_{2} s^{\prime}\left(\frac{t}{2}\right)^{s^{\prime}-1}+2 \cdot 2^{-1} s^{\prime}\left(s^{\prime}-1\right)\left(\frac{t}{2}\right)^{s^{\prime}-2}$ ) when $f=2^{-u_{0}-u_{t^{\prime}}}\left(\begin{array}{c}|\mathbf{u}| \\ \mathbf{u}\end{array}\right) \cdot z_{t, 0} u_{i}=2^{-u_{0}-u_{t^{\prime}}}\left(\begin{array}{c}|\mathbf{u}| \\ \mathbf{u}\end{array}\right) \cdot\left([s]_{2} u_{i}+2 u_{0} u_{i}\right)$ for some $1 \leqslant i \leqslant t^{\prime}-1$;

9. $[s]_{2} s^{\prime}\left(\frac{t}{2}\right)^{s^{\prime}-1}+s^{\prime}\left(s^{\prime}-1\right)\left(\frac{t}{2}\right) s^{s^{\prime}-2}$ (comes from $[s]_{2} \cdot 2 \cdot 2^{-1} s^{\prime}\left(\frac{t}{2}\right) s^{s^{\prime}-1}+2^{2} \cdot 2^{-2} s^{\prime}\left(s^{\prime}-\right.$ 1) $\left.\left(\frac{t}{2}\right)^{s^{\prime}-2}\right)$ when $f=2^{-u_{0}-u_{t^{\prime}}}\left(\begin{array}{c}|\mathbf{u}| \\ \mathbf{u}\end{array}\right) \cdot z_{t, 0} z_{t, t^{\prime}}=2^{-u_{0}-u_{t^{\prime}}}\left(\begin{array}{c}|\mathbf{u}| \\ \mathbf{u}\end{array}\right) \cdot\left([s]_{2} \cdot 2 u_{t^{\prime}}+2 u_{0} \cdot 2 u_{t^{\prime}}\right)$.

Finally we look at 'ordinary' cases, freely using $z_{t, 0}=2 u_{0}+[s]_{2}$.

1. $\left.\begin{array}{c}s^{\prime}+t^{\prime} \\ t^{\prime}\end{array}\right)$ when $f=1$;

2. $2\left(\begin{array}{c}s^{\prime}+t^{\prime} \\ t^{\prime}+2\end{array}\right)+\left(\begin{array}{c}s^{\prime}+t^{\prime} \\ t^{\prime}+1\end{array}\right)$ when $f=u_{i}^{2}=u_{i}\left(u_{i}-1\right)+u_{i}$ for some $1 \leqslant i \leqslant t^{\prime}$;

3. $\left(\begin{array}{c}s^{\prime}+t^{\prime} \\ t^{\prime}+2\end{array}\right)$ when $f=u_{i} u_{j}$ for some distinct $1 \leqslant i<j \leqslant t^{\prime}$.

4. $\frac{[s]_{2}^{2}\left(\begin{array}{c}s^{\prime}+t^{\prime} \\ t^{\prime}\end{array}\right)+\left(4+4[s]_{2}\right)\left(\begin{array}{c}s^{\prime}+t^{\prime} \\ t^{\prime}+1\end{array}\right)+8\left(\begin{array}{c}s^{\prime}+t^{\prime} \\ t^{\prime}+2\end{array}\right)}{4 u_{0}\left(u_{0}-1\right) \text { for some } 1 \leqslant i \leqslant t^{\prime} ;}$ when $f=z_{t, 0}^{2}=[s]_{2}^{2}+\left(4+4[s]_{2}\right) u_{0}+$ 
5. $[s]_{2}\left(\begin{array}{c}s^{\prime}+t^{\prime} \\ t^{\prime}+1\end{array}\right)+2\left(\begin{array}{c}s^{\prime}+t^{\prime} \\ t^{\prime}+2\end{array}\right)$ when $f=z_{t, 0} u_{i}=[s]_{2} u_{i}+2 u_{0} u_{i}$ for some $1 \leqslant i \leqslant t^{\prime}$.

Proof of "exponential" cases. We can mimic the methods in Corollary 4.8, using the exponential generating function $\exp \left(2^{-1} Z_{0} T\right) \prod_{i=1}^{t^{\prime}} \exp \left(Z_{i} T\right)$ when $t$ is odd, but instead the exponential generating function $\exp \left(2^{-1} Z_{0} T\right) \exp \left(2^{-1} Z_{t^{\prime}} T\right) \prod_{i=1}^{t^{\prime}-1} \exp \left(Z_{i} T\right)$ when $t$ is even.

Proof of "ordinary" cases. We can mimic the methods in Corollary 4.8, using the ordinary generating function $\prod_{i=1}^{t^{\prime}}\left(1-Z_{i} T\right)^{-1}$.

\subsubsection{Size of a self-conjugate $t$-core}

Lemma 4.10 (for $\mathcal{C}_{t}$ ) simplifies in $u$-coordinates for $\mathcal{D}_{t}$.

Lemma 6.14. Fix coprime $s, t \geqslant 1$ and $\lambda \in \mathcal{D}_{t}$. For convenience, set $s^{\prime}:=\lfloor s / 2\rfloor$ and $t^{\prime}:=\lfloor t / 2\rfloor$. We divide into cases based on the parity of $t$, but in both cases, the nonconstant (i.e. homogeneous quadratic) part of the quadratic, namely the polynomial given by $|\lambda|+\frac{1}{24}\left(t^{2}-1\right)$, has coefficients summing to 0 .

- If $t$ is odd, then the size $|\lambda|$ is given by a quadratic polynomial

$$
-\frac{1}{24}\left(t^{2}-1\right)+M_{2}\left(u_{1}, \ldots, u_{t^{\prime}}\right)+S_{2}\left(u_{1}, \ldots, u_{t^{\prime}}\right)+z_{t, 0} L_{1}\left(u_{1}, \ldots, u_{t^{\prime}}\right)+\frac{1}{24}\left(t^{2}-1\right) z_{t, 0}^{2}
$$

in $z_{t, 0}, u_{1}, \ldots, u_{t^{\prime}}$, where $S_{2}$ is a homogeneous quadratic with only 'square' terms, and with coefficient sum $\frac{1}{24}(t-2)\left(t^{2}-1\right) ; L_{1}$ is a homogeneous linear polynomial with coefficient sum $-2 \cdot \frac{1}{24}\left(t^{2}-1\right)$; and $M_{2}$ is the "leftover" homogeneous quadratic with only 'mixed' terms, and with coefficient sum $-\frac{1}{24}(t-3)\left(t^{2}-1\right)$.

- If $t$ is even, then the size $|\lambda|$ is given by a quadratic polynomial

$$
\begin{aligned}
-\frac{1}{24}\left(t^{2}-1\right) & +M_{2}\left(u_{1}, \ldots, u_{t^{\prime}-1}\right)+S_{2}\left(u_{1}, \ldots, u_{t^{\prime}-1}\right)+\frac{1}{24}\left(t^{2}-1\right)\left(z_{t, t^{\prime}}^{2}+z_{t, 0}^{2}\right) \\
& -\frac{1}{24}\left(t^{2}+2\right) z_{t, 0} z_{t, t^{\prime}}+z_{t, 0} L_{0}\left(u_{1}, \ldots, u_{t^{\prime}-1}\right)+z_{t, t^{\prime}} L_{t^{\prime}}\left(u_{1}, \ldots, u_{t^{\prime}-1}\right)
\end{aligned}
$$

in $z_{t, 0}, u_{1}, \ldots, u_{t^{\prime}-1}, z_{t, t^{\prime}}$, where $S_{2}$ is a homogeneous quadratic with only 'square' terms, and with coefficient sum $\frac{1}{24}(t-2)\left(t^{2}-2 t-2\right) ; L_{0}$ is a homogeneous linear polynomial with coefficient sum $-2 \cdot \frac{1}{24}\left(t^{2}-1\right)+\frac{1}{24}\left(t^{2}+2\right)=-\frac{1}{24}\left(t^{2}-4\right) ; L_{t^{\prime}}$ is a homogeneous linear polynomial with coefficient sum $2 \cdot \frac{1}{24}\left(t^{2}+2\right)$; and $M_{2}$ is the "leftover" homogeneous quadratic with with only 'mixed' terms, and with coefficient sum $-\frac{1}{24}\left(t^{3}-2 t^{2}+2 t+8\right)$.

Recap of $z$-coordinate preliminaries. Write $|\lambda|=-\frac{1}{24}\left(t^{2}-1\right)+\frac{1}{2 t} \cdot P$ for convenience. Since $\lambda \in \mathcal{D}_{t} \subseteq \mathcal{C}_{t}$ and $s, t$ are coprime, Equation (1) (from the proof of Lemma 4.10) gives the $a-$ to- $z$ translation $P:=\sum_{i=0}^{t-1}\left[a_{t, i}-\frac{t-1}{2}\right]^{2}=\sum_{\ell \in \mathbb{Z} / t \mathbb{Z}}\left(\sum_{j=0}^{t-1}\left(\frac{t-1}{2}-j\right) z_{t, j+\ell}\right)^{2}$, with $P(1, \ldots, 1)=$ 0 in the $z$-coordinates. Recall also from Lemma 4.10 that $\left[z_{t, i}^{2}\right] P=\sum_{j=0}^{t-1}\left(\frac{t-1}{2}-j\right)^{2}=$ $\frac{1}{12} t\left(t^{2}-1\right)$ for all $i \in \mathbb{Z} / t \mathbb{Z}$. 
We finish by breaking into cases based on parity of $t$ and using Proposition 6.11 (which applies to $\mathcal{D}_{t}$, not just $\left.\mathcal{D}_{s} \cap \mathcal{D}_{t}\right)$. For convenience, let $[x]_{t} \in\{0,1, \ldots, t-1\}$ denote the least nonnegative residue of $x(\bmod t)$.

Proof of Lemma 6.14 for odd $t$. In the $u$-coordinates (using $u_{i}=z_{t, i}=z_{t,-i}$ for $1 \leqslant i \leqslant$ $\frac{t-1}{2}=t^{\prime}$ ), the polynomial $P$ takes the form

$$
P=M\left(u_{1}, \ldots, u_{t^{\prime}}\right)+\sum_{i=1}^{t^{\prime}} \alpha_{i} u_{i}^{2}+\sum_{i=1}^{t^{\prime}} \beta_{i} u_{i} z_{t, 0}+\alpha_{0} z_{t, 0}^{2} .
$$

We make the following calculations, often suppressing $z_{i}:=z_{t, i}$.

- We have $\alpha_{0}=\left[z_{0}^{2}\right] P$ equal to $\sum_{j=0}^{t-1}\left(\frac{t-1}{2}-j\right)^{2}$ (which simplifies as $\left.\frac{1}{12} t\left(t^{2}-1\right)\right)$.

- To determine $\alpha_{i}$ (for $\left.1 \leqslant i \leqslant \frac{t-1}{2}\right)$, we evaluate $\alpha_{i}=\left[u_{i}^{2}\right] P=\left(\left[z_{i}^{2}\right] P+\left[z_{-i}^{2}\right] P+\right.$ $\left.\left[z_{i} z_{-i}\right] P\right)$ as $2 \cdot \frac{1}{12} t\left(t^{2}-1\right)+2 \sum_{\ell \in \mathbb{Z} / t \mathbb{Z}}\left(\frac{t-1}{2}-[i-\ell]_{t}\right)\left(\frac{t-1}{2}-[-i-\ell]_{t}\right)$, or $2 \cdot \frac{1}{12} t\left(t^{2}-1\right)+$ $2 \sum_{\ell \in \mathbb{Z} / t \mathbb{Z}}\left(\frac{t-1}{2}-[2 i+\ell]_{t}\right)\left(\frac{t-1}{2}-[\ell]_{t}\right)$. It follows that $\sum_{i=0}^{t^{\prime}} \alpha_{i}=\left[z_{0}^{2}\right] P+\frac{1}{2} \sum_{i=1}^{t-1}\left(\left[z_{i}^{2}\right] P+\right.$ $\left.\left[z_{-i}^{2}\right] P+\left[z_{i} z_{-i}\right] P\right)$ evaluates to

$$
(t-1) \cdot \frac{t\left(t^{2}-1\right)}{12}+\sum_{\ell \in \mathbb{Z} / t \mathbb{Z}} \sum_{i=0}^{t-1}\left(\frac{t-1}{2}-[\ell]_{t}\right)\left(\frac{t-1}{2}-[2 i+\ell]_{t}\right) .
$$

But $\operatorname{gcd}(2, t)=1$, so $\sum_{i=0}^{t-1}\left(\frac{t-1}{2}-[2 i+\ell]_{t}\right)=0$ for each $\ell \in \mathbb{Z} / t \mathbb{Z}$. Thus $\sum_{i=0}^{t^{\prime}} \alpha_{i}=$ $(t-1) \cdot \frac{1}{12} t\left(t^{2}-1\right)$, and $\sum_{i=1}^{t^{\prime}} \alpha_{i}=(t-2) \cdot \frac{1}{12} t\left(t^{2}-1\right)$.

- Similarly, we expand (for $\left.1 \leqslant i \leqslant \frac{t-1}{2}\right) \beta_{i}=\left(\left[z_{0} z_{i}\right]+\left[z_{0} z_{-i}\right]\right) P=4 \sum_{\ell \in \mathbb{Z} / t \mathbb{Z}}\left(\frac{t-1}{2}-\right.$ $\left.[i+\ell]_{t}\right)\left(\frac{t-1}{2}-[\ell]_{t}\right)$, so that $\sum_{i=1}^{t^{\prime}} \beta_{i}=2 \sum_{\ell \in \mathbb{Z} / t \mathbb{Z}}\left(\frac{t-1}{2}-[\ell]_{t}\right) \sum_{i=1}^{t-1}\left(\frac{t-1}{2}-[i+\ell]_{t}\right)=$ $2 \sum_{\ell}-\left(\frac{t-1}{2}-[\ell]_{t}\right)^{2}=-2 \cdot \frac{1}{12} t\left(t^{2}-1\right)$.

Finally, $P(1, \ldots, 1)=0$ gives $M(1, \ldots, 1)=-(t-3) \cdot \frac{1}{12} t\left(t^{2}-1\right)$, and substituting $P$ into $|\lambda|=-\frac{1}{24}\left(t^{2}-1\right)+\frac{1}{2 t} P$ finishes the job.

Proof of Lemma 6.14 for even $t$. In the $u$-coordinates (using $u_{i}=z_{t, i}=z_{t,-i}$ for $1 \leqslant i \leqslant$ $\left.\frac{t}{2}-1=t^{\prime}-1\right)$, the polynomial $P$ takes the form

$$
P=M\left(u_{1}, \ldots, u_{t^{\prime}-1}\right)+\sum_{i=1}^{t^{\prime}-1} \gamma_{i} u_{i} z_{t, t^{\prime}}+\sum_{i=1}^{t^{\prime}-1} \alpha_{i} u_{i}^{2}+\sum_{i=1}^{t^{\prime}-1} \beta_{i} u_{i} z_{t, 0}+\alpha_{0} z_{t, 0}^{2}+\beta_{t^{\prime}} z_{t, 0} z_{t^{\prime}}+\alpha_{t^{\prime}} z_{t, t^{\prime}}^{2}
$$

We make the following calculations, often suppressing $z_{i}:=z_{t, i}$.

- We have $\alpha_{0}=\left[z_{t, 0}^{2}\right] P$ and $\alpha_{t^{\prime}}=\left[z_{t, t^{\prime}}^{2}\right] P$ both equal to $\sum_{j=0}^{t-1}\left(\frac{t-1}{2}-j\right)^{2}$ (which simplifies as $\left.\frac{1}{12} t\left(t^{2}-1\right)\right)$. 
- For $1 \leqslant i \leqslant t^{\prime}-1$, we evaluate $\alpha_{i}=\left[u_{i}^{2}\right] P=2 \cdot \frac{1}{12} t\left(t^{2}-1\right)+2 \sum_{\ell \in \mathbb{Z} / t \mathbb{Z}}\left(\frac{t-1}{2}-\right.$ $\left.[\ell]_{t}\right)\left(\frac{t-1}{2}-[2 i+\ell]_{t}\right)$ as in the odd $t$ case. It follows that $\sum_{i=0}^{t^{\prime}} \alpha_{i}=\left[z_{0}^{2}\right] P+\left[z_{t^{\prime}}^{2}\right] P+$ $\frac{1}{2} \sum_{0<\left|i-t^{\prime}\right|<t^{\prime}}\left(\left[z_{i}^{2}\right] P+\left[z_{-i}^{2}\right] P+\left[z_{i} z_{-i}\right] P\right)$ evaluates to

$$
(t-2) \cdot \frac{t\left(t^{2}-1\right)}{12}+\sum_{\ell \in \mathbb{Z} / t \mathbb{Z}}\left(\frac{t-1}{2}-[\ell]_{t}\right) \sum_{i=0}^{t-1}\left(\frac{t-1}{2}-[2 i+\ell]_{t}\right) .
$$

Note that $\sum_{i=0}^{t-1}\left(\frac{t-1}{2}-[2 i+\ell]_{t}\right)$ depends only on the parity of the residue class $\ell$ $(\bmod t)$; it equals $\frac{t-1}{2}-2(0+2+\cdots+(t-2))=(1+3+\cdots+(t-1))-(0+2+\cdots+(t-$ $2))=t^{\prime}$ if $\ell$ is even, and $-t^{\prime}$ if $\ell$ is odd. Thus $\sum_{\ell \in \mathbb{Z} / t \mathbb{Z}}\left(\frac{t-1}{2}-[\ell]_{t}\right) \sum_{i=0}^{t-1}\left(\frac{t-1}{2}-[2 i+\ell]_{t}\right)$ evaluates to $\sum_{j=0}^{t^{\prime}-1}\left(\frac{t-1}{2}-2 j\right) t^{\prime}+\left(\frac{t-1}{2}-2 j-1\right)\left(-t^{\prime}\right)=\sum_{j=0}^{t^{\prime}-1} 1 \cdot t^{\prime}=\frac{1}{4} t^{2}$, whence $\sum_{i=0}^{t^{\prime}} \alpha_{i}=(t-2) \cdot \frac{1}{12} t\left(t^{2}-1\right)+\frac{1}{4} t^{2}$, and $\sum_{i=1}^{t^{\prime}-1} \alpha_{i}=(t-4) \cdot \frac{1}{12} t\left(t^{2}-1\right)+\frac{1}{4} t^{2}=$ $\frac{1}{12} t(t-2)\left(t^{2}-2 t-2\right)$.

- Next, $\beta_{t^{\prime}}=\left[z_{0} z_{t^{\prime}}\right] P=2 \sum_{\ell \in \mathbb{Z} / t \mathbb{Z}}\left(\frac{t-1}{2}-\left[t^{\prime}+\ell\right]_{t}\right)\left(\frac{t-1}{2}-[\ell]_{t}\right)$, which simplifies to $2 \sum_{j=0}^{t^{\prime}-1}\left(\frac{t-1}{2}-j\right)\left(\frac{t-1}{2}-j-t^{\prime}\right)+\left(\frac{t-1}{2}-j-t^{\prime}\right)\left(\frac{t-1}{2}-j\right)=-\frac{1}{3} t^{\prime}\left(2 t^{\prime 2}+1\right)=-\frac{1}{12} t\left(t^{2}+2\right)$.

- Similarly, we expand (for $\left.1 \leqslant i \leqslant t^{\prime}-1\right) \beta_{i}=\left(\left[z_{0} z_{i}\right]+\left[z_{0} z_{-i}\right]\right) P=4 \sum_{\ell \in \mathbb{Z} / t \mathbb{Z}}\left(\frac{t-1}{2}-[i+\right.$ $\left.\ell]_{t}\right)\left(\frac{t-1}{2}-[\ell]_{t}\right)$, so that $\sum_{i=1}^{t^{\prime}} \beta_{i}=2 \sum_{\ell}\left(\frac{t-1}{2}-[\ell]_{t}\right) \sum_{i=1}^{t-1}\left(\frac{t-1}{2}-[i+\ell]_{t}\right)=2 \sum_{\ell}-\left(\frac{t-1}{2}-\right.$ $\left.[\ell]_{t}\right)^{2}=-2 \cdot \frac{1}{12} t\left(t^{2}-1\right)$. It follows that $\sum_{i=1}^{t^{\prime}-1} \beta_{i}=-2 \cdot \frac{1}{12} t\left(t^{2}-1\right)-\beta_{t^{\prime}}=-2$. $\frac{1}{12} t\left(t^{2}-1\right)+\frac{1}{12} t\left(t^{2}+2\right)=-\frac{1}{12} t\left(t^{2}-4\right)$.

- It remains to compute, for $1 \leqslant i \leqslant t^{\prime}-1$, the coefficient $\gamma_{i}=\left(\left[z_{t^{\prime}} z_{i}\right]+\left[z_{t^{\prime}} z_{-i}\right]\right) P=$ $4 \sum_{\ell \in \mathbb{Z} / t \mathbb{Z}}\left(\frac{t-1}{2}-\left[t^{\prime}+i+\ell\right]_{t}\right)\left(\frac{t-1}{2}-[\ell]_{t}\right)$, so that $\beta_{t^{\prime}}+\sum_{i=1}^{t^{\prime}-1} \gamma_{i}=2 \sum_{\ell \in \mathbb{Z} / t \mathbb{Z}}\left(\frac{t-1}{2}-\right.$ $\left.[\ell]_{t}\right) \sum_{i=1}^{t-1}\left(\frac{t-1}{2}-\left[t^{\prime}+i+\ell\right]_{t}\right)=2 \sum_{\ell \in \mathbb{Z} / t \mathbb{Z}}-\left(\frac{t-1}{2}-[\ell]_{t}\right)\left(\frac{t-1}{2}-\left[t^{\prime}+\ell\right]_{t}\right)=-\beta_{t^{\prime}}$. Thus $\sum_{i=1}^{t^{\prime}-1} \gamma_{i}=-2 \beta_{t^{\prime}}=2 \cdot \frac{1}{12} t\left(t^{2}+2\right)$.

Finally, $P(1, \ldots, 1)=0$ gives $M(1, \ldots, 1)=-\frac{1}{12} t\left(t^{3}-2 t^{2}+2 t+8\right)$, and substituting $P$ into $|\lambda|=-\frac{1}{24}\left(t^{2}-1\right)+\frac{1}{2 t} P$ finishes the job.

\subsection{Proofs of self-conjugate conjectures}

This section is the self-conjugate analog of Section 5, addressing Armstrong's and Fayers' self-conjugate conjectures.

Proof of Theorem 1.2 by direct computation for odd $t$. We use $u$-coordinates. For convenience, let $s^{\prime}=\lfloor s / 2\rfloor$ and $t^{\prime}=\lfloor t / 2\rfloor$, and let $[s]_{2}:=s-2 s^{\prime} \in\{0,1\}$. By Proposition 6.13, the denominator is just $\sum_{U_{t}(s)} 1=\left(\begin{array}{c}t^{\prime}+s^{\prime} \\ t^{\prime}\end{array}\right)$.

For odd $t$, Lemma 6.14 says the size $|\lambda|$ is given by a quadratic polynomial

$$
-\frac{1}{24}\left(t^{2}-1\right)+M_{2}\left(u_{1}, \ldots, u_{t^{\prime}}\right)+S_{2}\left(u_{1}, \ldots, u_{t^{\prime}}\right)+z_{t, 0} L_{1}\left(u_{1}, \ldots, u_{t^{\prime}}\right)+\frac{1}{24}\left(t^{2}-1\right) z_{t, 0}^{2}
$$

in $z_{t, 0}, u_{1}, \ldots, u_{t^{\prime}}$, where $M_{2}$ is a homogeneous quadratic with only 'mixed' terms, and coefficient sum $-\frac{1}{24}(t-3)\left(t^{2}-1\right) ; S_{2}$ is a homogeneous quadratic with only 'square' 
terms, and coefficient sum $\frac{1}{24}(t-2)\left(t^{2}-1\right)$; and $L_{1}$ is a homogeneous linear polynomial with coefficient sum $-2 \cdot \frac{1}{24}\left(t^{2}-1\right)$. Then by Proposition 6.13 , the numerator $\sum_{U_{t}(s)}|\lambda|$ evaluates to

$$
\begin{aligned}
& -\frac{t^{2}-1}{24} \cdot\left(\begin{array}{c}
N \\
t^{\prime}
\end{array}\right)-\frac{(t-3)\left(t^{2}-1\right)}{24} \cdot\left(\begin{array}{c}
N \\
t^{\prime}+2
\end{array}\right)+\frac{(t-2)\left(t^{2}-1\right)}{24}\left[2\left(\begin{array}{c}
N \\
t^{\prime}+2
\end{array}\right)+\left(\begin{array}{c}
N \\
t^{\prime}+1
\end{array}\right)\right] \\
& -2 \frac{t^{2}-1}{24}\left[[s]_{2}\left(\begin{array}{c}
N \\
t^{\prime}+1
\end{array}\right)+2\left(\begin{array}{c}
N \\
t^{\prime}+2
\end{array}\right)\right] \\
& +\frac{t^{2}-1}{24}\left[[s]_{2}^{2}\left(\begin{array}{c}
N \\
t^{\prime}
\end{array}\right)+\left(4+4[s]_{2}\right)\left(\begin{array}{c}
N \\
t^{\prime}+1
\end{array}\right)+8\left(\begin{array}{c}
N \\
t^{\prime}+2
\end{array}\right)\right],
\end{aligned}
$$

where we have suppressed $N:=s^{\prime}+t^{\prime}$. Collecting terms, and using $\left(\begin{array}{c}N \\ t^{\prime}+1\end{array}\right)=\frac{s^{\prime}}{t^{\prime}+1}\left(\begin{array}{c}N \\ t^{\prime}\end{array}\right)$ and $\left(\begin{array}{c}N \\ t^{\prime}+2\end{array}\right)=\frac{s^{\prime}-1}{t^{\prime}+2}\left(\begin{array}{c}N \\ t^{\prime}+1\end{array}\right)$ (viewed as polynomial identities in $s^{\prime}$, for fixed $t^{\prime} \geqslant 0$ ), the numerator becomes

$$
\begin{aligned}
\frac{t^{2}-1}{24}\left[\left(-1+[s]_{2}^{2}\right)\left(\begin{array}{l}
N \\
t^{\prime}
\end{array}\right)+[-(t-3)+2(t-2)-2 \cdot 2+8]\left(\begin{array}{c}
N \\
t^{\prime}+2
\end{array}\right)\right. \\
\left.+\left[(t-2)-2[s]_{2}+\left(4+4[s]_{2}\right)\right]\left(\begin{array}{c}
N \\
t^{\prime}+1
\end{array}\right)\right] \\
\quad=\frac{t^{2}-1}{24}\left(\begin{array}{l}
N \\
t^{\prime}
\end{array}\right)\left[\left(-1+[s]_{2}^{2}\right)+\frac{4 s^{\prime}\left(s^{\prime}-1\right)}{t+1}+\left[t+2+2[s]_{2}\right] \frac{2 s^{\prime}}{t+1}\right],
\end{aligned}
$$

where we have used $t^{\prime}+2=\frac{t-1}{2}+2=\frac{t+3}{2}$ to simplify. Factoring $t^{2}-1=(t-1)(t+1)$ and multiplying through by $t+1$ transforms the numerator expression to the product of $\frac{1}{24}(t-1)\left(\begin{array}{l}N \\ t^{\prime}\end{array}\right)$ with

$$
\left(-1+[s]_{2}^{2}\right)(t+1)+4 s^{\prime 2}+4[s]_{2} s^{\prime}+2 t s^{\prime}=-t-1+\left(2 s^{\prime}+[s]_{2}\right)^{2}+\left([s]_{2}^{2}+2 s^{\prime}\right) t .
$$

But $[s]_{2}^{2}=[s]_{2}\left(\right.$ and $\left.2 s^{\prime}+[s]_{2}=s\right)$, so the product evaluates to $\frac{1}{24}(t-1)\left(\begin{array}{l}N \\ t^{\prime}\end{array}\right)\left(-t-1+s^{2}+s t\right)$, which factors as $\frac{1}{24}(t-1)\left(\begin{array}{l}N \\ t^{\prime}\end{array}\right)(s-1)(s+t+1)$. Dividing numerator by denominator yields the desired ratio of $\frac{1}{24}(s-1)(t-1)(s+t+1)$.

Proof of Theorem 1.2 for even $t$. If $t$ is even, then $s$ is odd. But the un-weighted problem is symmetric in $s, t$, so swapping the roles of $s, t$ in the previous proof establishes the claim.

The same techniques prove Fayers' self-conjugate conjecture.

Proof of Theorem 1.4 for odd $t$. We use $u$-coordinates. For convenience, let $s^{\prime}=\lfloor s / 2\rfloor$ and $t^{\prime}=\lfloor t / 2\rfloor$, and let $[s]_{2}:=s-2 s^{\prime} \in\{0,1\}$. By Proposition 6.12 and Proposition 6.13, the denominator times $s^{\prime}$ ! is just $\sum_{U_{t}(s)} 2^{-u_{0}}\left(\begin{array}{c}|\mathbf{u}| \\ \mathbf{u}\end{array}\right)=\left(\frac{t}{2}\right)^{s^{\prime}}$ where $\mathbf{u}=\left(u_{0}, \ldots, u_{t^{\prime}}\right)$.

For odd $t$, Lemma 6.14 says the size $|\lambda|$ is given by a quadratic polynomial

$$
-\frac{1}{24}\left(t^{2}-1\right)+M_{2}\left(u_{1}, \ldots, u_{t^{\prime}}\right)+S_{2}\left(u_{1}, \ldots, u_{t^{\prime}}\right)+z_{t, 0} L_{1}\left(u_{1}, \ldots, u_{t^{\prime}}\right)+\frac{1}{24}\left(t^{2}-1\right) z_{t, 0}^{2}
$$


in $z_{t, 0}, u_{1}, \ldots, u_{t^{\prime}}$, where $M_{2}$ is a homogeneous quadratic with only 'mixed' terms, and coefficient sum $-\frac{1}{24}(t-3)\left(t^{2}-1\right) ; S_{2}$ is a homogeneous quadratic with only 'square' terms, and coefficient sum $\frac{1}{24}(t-2)\left(t^{2}-1\right)$; and $L_{1}$ is a homogeneous linear polynomial with coefficient sum $-2 \cdot \frac{1}{24}\left(t^{2}-1\right)$. Then by Propositions 6.12 and 6.13 , the numerator times $s^{\prime}$ !, i.e. the sum $\sum_{U_{t}(s)} 2^{-u_{0}}\left(\begin{array}{c}|\mathbf{u}| \\ \mathbf{u}\end{array}\right)|\lambda|$, evaluates to

$$
\begin{aligned}
& -\frac{1}{24}\left(t^{2}-1\right) \cdot N^{s^{\prime}}-\frac{1}{24}(t-3)\left(t^{2}-1\right) \cdot s^{\prime}\left(s^{\prime}-1\right) N^{s^{\prime}-2} \\
& +\frac{1}{24}(t-2)\left(t^{2}-1\right)\left[s^{\prime}\left(s^{\prime}-1\right) N^{s^{\prime}-2}+s^{\prime} N^{s^{\prime}-1}\right] \\
& -2 \cdot \frac{1}{24}\left(t^{2}-1\right)\left([s]_{2} s^{\prime} N^{s^{\prime}-1}+s^{\prime}\left(s^{\prime}-1\right) N^{s^{\prime}-2}\right) \\
& +\frac{1}{24}\left(t^{2}-1\right)\left([s]_{2}^{2} N^{s^{\prime}}+\left(2+2[s]_{2}\right) s^{\prime} N^{s^{\prime}-1}+s^{\prime}\left(s^{\prime}-1\right) N^{s^{\prime}-2}\right),
\end{aligned}
$$

where we have suppressed $N:=\frac{t}{2}$. Collecting terms, we have

- $N^{s^{\prime}}$ coefficient $-\frac{1}{24}\left(t^{2}-1\right)+\frac{1}{24}\left(t^{2}-1\right)[s]_{2}^{2}$;

- $s^{\prime} N^{s^{\prime}-1}$ coefficient $\frac{1}{24}\left(t^{2}-1\right)\left[(t-2)-2[s]_{2}+\left(2+2[s]_{2}\right)\right]$, which is just $\frac{1}{24}\left(t^{2}-1\right) \cdot t$;

- $s^{\prime}\left(s^{\prime}-1\right) N^{s^{\prime}-2}$ coefficient $-\frac{1}{24}(t-3)\left(t^{2}-1\right)+\frac{1}{24}(t-2)\left(t^{2}-1\right)-2 \cdot \frac{1}{24}\left(t^{2}-1\right)+\frac{1}{24}\left(t^{2}-1\right)$, or more conceptually, the sum of coefficients of the polynomial $|\lambda|+\frac{1}{24}\left(t^{2}-1\right)$, which is 0 as noted at the case-free beginning of Lemma 6.14,

so that the numerator is $N^{s^{\prime}} \cdot \frac{1}{24}\left(t^{2}-1\right)\left(-1+[s]_{2}^{2}+t \cdot \frac{s^{\prime}}{N}\right)$, which simplifies, via $[s]_{2}^{2}=[s]_{2}=$ $s-2 s^{\prime}$, as $N^{s^{\prime}} \cdot \frac{1}{24}\left(t^{2}-1\right)(s-1)$. Finally, dividing numerator (times $s^{\prime}$ !) by denominator (times $s^{\prime}$ !) yields the desired ratio of $\frac{1}{24}(s-1)\left(t^{2}-1\right)$.

Proof of Theorem 1.4 for even $t$. We use $u$-coordinates. For convenience, let $s^{\prime}=\lfloor s / 2\rfloor$ and $t^{\prime}=\lfloor t / 2\rfloor$, and let $[s]_{2}:=s-2 s^{\prime} \in\{0,1\}$. By Proposition 6.12 and Proposition 6.13, the denominator times $s^{\prime}$ ! is just $\sum_{U_{t}(s)} 2^{-u_{0}}\left(\begin{array}{c}|\mathbf{u}| \\ \mathbf{u}\end{array}\right)=\left(\frac{t}{2}\right)^{s^{\prime}}$.

For even $t$, Lemma 6.14 says the size $|\lambda|$ is given by a quadratic polynomial

$$
\begin{aligned}
-\frac{1}{24}\left(t^{2}-1\right) & +M_{2}\left(u_{1}, \ldots, u_{t^{\prime}-1}\right)+S_{2}\left(u_{1}, \ldots, u_{t^{\prime}-1}\right)+\frac{1}{24}\left(t^{2}-1\right)\left(z_{t, t^{\prime}}^{2}+z_{t, 0}^{2}\right) \\
& -\frac{1}{24}\left(t^{2}+2\right) z_{t, 0} z_{t, t^{\prime}}+z_{t, 0} L_{0}\left(u_{1}, \ldots, u_{t^{\prime}-1}\right)+z_{t, t^{\prime}} L_{t^{\prime}}\left(u_{1}, \ldots, u_{t^{\prime}-1}\right)
\end{aligned}
$$

in $z_{t, 0}, u_{1}, \ldots, u_{t^{\prime}-1}, z_{t, t^{\prime}}$, where $M_{2}$ is a homogeneous quadratic with with only 'mixed' terms, and with coefficient sum $-\frac{1}{24}\left(t^{3}-2 t^{2}+2 t+8\right) ; S_{2}$ is a homogeneous quadratic with only 'square' terms, and coefficient sum $\frac{1}{24}(t-2)\left(t^{2}-2 t-2\right) ; L_{0}$ is a homogeneous linear polynomial with coefficient sum $-2 \cdot \frac{1}{24}\left(t^{2}-1\right)+\frac{1}{24}\left(t^{2}+2\right)=-\frac{1}{24}\left(t^{2}-4\right)$; and $L_{t^{\prime}}$ is a homogeneous linear polynomial with coefficient sum $2 \cdot \frac{1}{24}\left(t^{2}+2\right)$. Then by Propositions 6.12 and 6.13 , the numerator times $s^{\prime}$ !, i.e. the sum $\sum_{U_{t}(s)} 2^{-u_{0}}\left(\begin{array}{c}|\mathbf{u}| \\ \mathbf{u}\end{array}\right)|\lambda|$, is just the sum of the following terms:

- $-\frac{1}{24}\left(t^{2}-1\right) \cdot N^{s^{\prime}}$ (from constant term);

- $-\frac{1}{24}\left(t^{3}-2 t^{2}+2 t+8\right) \cdot s^{\prime}\left(s^{\prime}-1\right) N^{s^{\prime}-2}\left(\right.$ from $\left.M_{2}\right)$; 
- $\frac{1}{24}(t-2)\left(t^{2}-2 t-2\right) \cdot\left[s^{\prime}\left(s^{\prime}-1\right) N^{s^{\prime}-2}+s^{\prime} N^{s^{\prime}-1}\right]\left(\right.$ from $\left.S_{2}\right)$;

- $\frac{1}{24}\left(t^{2}-1\right) \cdot\left[s^{\prime}\left(s^{\prime}-1\right) N^{s^{\prime}-2}+2 s^{\prime} N^{s^{\prime}-1}\right]\left(\right.$ from $\left.z_{t, t^{\prime}}^{2}\right)$;

- $\frac{1}{24}\left(t^{2}-1\right) \cdot\left[[s]_{2}^{2} N^{s^{\prime}}+\left(2+2[s]_{2}\right) s^{\prime} N^{s^{\prime}-1}+s^{\prime}\left(s^{\prime}-1\right) N^{s^{\prime}-2}\right]\left(\right.$ from $\left.z_{t, 0}^{2}\right)$;

- $-\frac{1}{24}\left(t^{2}+2\right) \cdot\left[[s]_{2} s^{\prime} N^{s^{\prime}-1}+s^{\prime}\left(s^{\prime}-1\right) N^{s^{\prime}-2}\right]\left(\right.$ from $\left.z_{t, 0} z_{t, t^{\prime}}\right)$;

- $-\frac{1}{24}\left(t^{2}-4\right) \cdot\left[[s]_{2} s^{\prime} N^{s^{\prime}-1}+s^{\prime}\left(s^{\prime}-1\right) N^{s^{\prime}-2}\right]\left(\right.$ from $\left.z_{t, 0} L_{0}\right)$;

- $2 \cdot \frac{1}{24}\left(t^{2}+2\right) \cdot s^{\prime}\left(s^{\prime}-1\right) N^{s^{\prime}-2}\left(\right.$ from $\left.z_{t, t^{\prime}} L_{t^{\prime}}\right)$,

where we have suppressed $N:=\frac{t}{2}$. Collecting terms, we have

- $N^{s^{\prime}}$ coefficient $-\frac{1}{24}\left(t^{2}-1\right)+\frac{1}{24}\left(t^{2}-1\right)[s]_{2}^{2}$, which is 0 since $s$ is odd;

- $s^{\prime} N^{s^{\prime}-1}$ coefficient $\frac{1}{24}(t-2)\left(t^{2}-2 t-2\right)+2 \cdot \frac{1}{24}\left(t^{2}-1\right)+\frac{1}{24}\left(t^{2}-1\right)\left(2+2[s]_{2}\right)-$ $[s]_{2} \frac{1}{24}\left(t^{2}+2\right)-\frac{1}{24}\left(t^{2}-4\right)$, which is $\frac{1}{24} t\left(t^{2}+2\right)$ since $s$ is odd;

- $s^{\prime}\left(s^{\prime}-1\right) N^{s^{\prime}-2}$ coefficient $-\frac{1}{24}\left(t^{3}-2 t^{2}+2 t+8\right)+\frac{1}{24}(t-2)\left(t^{2}-2 t-2\right)+\frac{1}{24}\left(t^{2}-\right.$ $1)+\frac{1}{24}\left(t^{2}-1\right)-\frac{1}{24}\left(t^{2}+2\right)-\frac{1}{24}\left(t^{2}-4\right)+2 \frac{1}{24}\left(t^{2}+2\right)$, or more conceptually, the sum of coefficients of the polynomial $|\lambda|+\frac{1}{24}\left(t^{2}-1\right)$, which is 0 as noted at the case-free beginning of Lemma 6.14 .

Thus the numerator times $s^{\prime}$ ! is $s^{\prime} N^{s^{\prime}-1} \cdot \frac{1}{24} t\left(t^{2}+2\right)=\frac{s-1}{2} N^{s^{\prime}} \cdot 2 \cdot \frac{1}{24}\left(t^{2}+2\right)=N^{s^{\prime}} \cdot \frac{1}{24}(s-$ $1)\left(t^{2}+2\right)$, where we have used $s^{\prime}=\frac{s-1}{2}$ (as $s$ must be odd). Dividing numerator (times $\left.s^{\prime} !\right)$ by denominator (times $\left.s^{\prime} !\right)$ yields the desired ratio of $\frac{1}{24}(s-1)\left(t^{2}+2\right)$.

\section{Counting $(m, m+d, m+2 d)$-cores}

We give two cyclic shifts proofs of Theorem 1.6, using different $z$-coordinate-based parameterizations of $\mathcal{C}_{m} \cap \mathcal{C}_{m+d} \cap \mathcal{C}_{m+2 d}$, which may generalize in different ways.

Remark 7.1. Explicitly, the proofs differ in that the first "symmetric proof" views $(m, m+$ $d, m+2 d)$-cores as $(m+d)$-cores that are also $(m, m+2 d)$-cores, while the second "asymmetric proof" views $(m, m+d, m+2 d)$-cores as $(m, m+d)$-cores that are also $(m+2 d)$-cores. The proofs both use (the change of variables from) Proposition 4.2 for different choices of coprime $s, t \geqslant 1$, but with $s$ only a "purely algebraic parameter" in the first proof.

Our first proof uses the extension of Proposition 4.2 for general $t$-cores, with $t=m+d$.

Symmetric proof. Let $(s, t)=(d, m+d)$, so $s, t \geqslant 1$ are coprime. By Lemma 3.1, a $t$-core $\lambda$ lies in $\mathcal{C}_{t-d}=\mathcal{C}_{m}$ if and only if $a_{t, i} \geqslant a_{t, i-d}-(t-d)$ for all $i \in \mathbb{Z} / t \mathbb{Z}$, and $\lambda \in \mathcal{C}_{t+d}=\mathcal{C}_{m+2 d}$ if and only if $a_{t, i} \geqslant a_{t, i+d}-(t+d)$ for all $i$. Thus $\lambda \in \mathcal{C}_{t}$ is a $(t-d, t, t+d)=(m, m+d, m+2 d)$-core if and only if

$$
t \geqslant a_{t, i}-\left[a_{t, i+d}-d\right] \geqslant-t
$$


for all $i{ }^{2}$ By Proposition 4.2 (applied to $(s, t)=(d, m+d)$ ) parameterizing $\mathcal{C}_{t}=\mathcal{C}_{m+d}$, these inequalities translate in $z_{t}$-coordinates - after division by $t$ - to $1 \geqslant z_{t, j} \geqslant-1$ (i.e. $\left.z_{t, j} \in\{-1,0,1\}\right)$, along with the usual $\sum_{j \in \mathbb{Z} / t \mathbb{Z}} z_{t, j}=s=d ; z_{t, j} \equiv 0(\bmod 1)$; and $\sum_{j \in \mathbb{Z} / t \mathbb{Z}} j z_{t, j} \equiv 0(\bmod t)$.

A cyclic shifts argument analogous to Proposition 4.7 yields

$$
\sum_{\left(z_{t, j}\right) \in \mathcal{C}_{m} \cap \mathcal{C}_{m+d} \cap \mathcal{C}_{m+2 d}} 1=\frac{1}{t} \sum_{\substack{x_{j} \in\{-1,0,1\} \\
\sum_{j \in \mathbb{Z} / t \mathbb{Z}} x_{j}=d}} 1=\frac{1}{t} \sum_{i=0}^{\lfloor(t-d) / 2\rfloor}\left(\begin{array}{c}
t \\
i, i+d, t-(2 i+d)
\end{array}\right),
$$

where in the last step we count valid sequences $\left(x_{j}\right)_{j \in \mathbb{Z} / t \mathbb{Z}} \in\{-1,0,1\}^{t}$ by the numbers $i, i+d, t-(2 i+d)$ of appearances of $-1,+1,0$, respectively. Substituting in $t=m+d$ completes the proof.

Our second proof uses the more familiar form of Proposition 4.2, for $(s, t)$-cores.

Asymmetric proof. Let $(s, t)=(m+d, m)$, so $s, t \geqslant 1$ are coprime. By Lemma 3.1, a $t$-core $\lambda$ lies in $\mathcal{C}_{s}=\mathcal{C}_{m+d}$ if and only if $a_{t, i} \geqslant a_{t, i+s}-s$ for all $i \in \mathbb{Z} / t \mathbb{Z}$, and $\lambda \in$ $\mathcal{C}_{s+d}=\mathcal{C}_{m+2 d}=\mathcal{C}_{2 s-t}$ if and only if $a_{t, i} \geqslant a_{t, i+2 s}-(2 s-t)$ for all $i$. Thus $\lambda \in \mathcal{C}_{t}$ is a $(t, s, 2 s-t)=(m, m+d, m+2 d)$-core if and only if $a_{t, i}-\left[a_{t, i+s}-s\right] \geqslant 0$ and $a_{t, i}-\left[a_{t, i+2 s}-2 s\right] \geqslant t$ for all $i$. By Proposition 4.2 (applied to $(s, t)=(m+d, m)$ ) parameterizing $\mathcal{C}_{s} \cap \mathcal{C}_{t}=\mathcal{C}_{m+d} \cap \mathcal{C}_{m}$, these inequalities translate in $z_{t}$-coordinates - after division by $t$ - to $z_{t, j}+z_{t, j+1} \geqslant 1$, along with the usual $z_{t, j} \geqslant 0 ; \sum_{j \in \mathbb{Z} / t \mathbb{Z}} z_{t, j}=s=m+d$; $z_{t, j} \equiv 0(\bmod 1) ;$ and $\sum_{j \in \mathbb{Z} / t \mathbb{Z}} j z_{t, j} \equiv 0(\bmod t)$.

A cyclic shifts argument analogous to Proposition 4.7 yields

$$
\sum_{\left(z_{t, j}\right) \in \mathcal{C}_{m} \cap \mathcal{C}_{m+d} \cap \mathcal{C}_{m+2 d}} 1=\frac{1}{t} \sum_{\substack{x_{j}, x_{j}+x_{j+1}-1 \geqslant 0 \\
\sum_{j \in \mathbb{Z} / t \mathbb{Z}} x_{j}=s}} 1=\frac{1}{t} \sum_{i=0}^{\lfloor t / 2\rfloor} \frac{t}{t-i}\left(\begin{array}{c}
t-i \\
i
\end{array}\right) \cdot\left(\begin{array}{c}
s-1 \\
(t-i)-1
\end{array}\right)
$$

where in the last step we count valid sequences $\left(x_{j}\right)_{j \in \mathbb{Z} / t \mathbb{Z}}$ by first choosing the $i$ pairwise non-neighboring 0 terms, and then the remaining $t-i$ positive integers summing to $s$. There are $\frac{t}{t-i}\left(\begin{array}{c}t-i \\ i\end{array}\right)$ ways to choose the $i$ zero-positions (we can double-count pairs $(S, \alpha)$ with $S \subseteq \mathbb{Z} / t \mathbb{Z}$ the set of $i$ zero-positions, and $\alpha \notin S$ some non-zero-position), and $\left(\begin{array}{c}s-1 \\ (t-i)-1\end{array}\right)$ ways to choose the $t-i$ positive integers. To finish, we note $\frac{1}{t-i}\left(\begin{array}{c}t-i \\ i\end{array}\right)\left(\begin{array}{c}s-1 \\ t-i-1\end{array}\right)=\frac{1}{s}\left(\begin{array}{c}s \\ i, s-t+i, t-2 i\end{array}\right)$ and then substitute in $t=m$ and $s=m+d$.

Remark 7.2. Using either parameterization and mimicking the proof of Theorems 1.1 (in particular the cyclic shifts), one could evaluate the sum of the sizes of $(m, m+d, m+2 d)$ cores. Mimicking Section 6, one could also study self-conjugate analogs for $\mathcal{D}_{m} \cap \mathcal{D}_{m+d} \cap$ $\mathcal{D}_{m+2 d}$. Also, in principle, one could probably carry much of this over to $(m, m+d, \ldots, m+$

\footnotetext{
${ }^{2}$ As described in Remark 3.5, Johnson essentially observed that the $d$-hooks in $\lambda$ correspond to the indices $i \in \mathbb{Z} / t \mathbb{Z}$ satisfying $a_{t, i}-\left[a_{t, i+d}-d\right]=-t$.
} 
$k d$ )-cores (for $k \geqslant 3$ ), or even to more general multiple cores, with the computational messiness growing with the complexity of the set of avoided hook lengths. However, it would likely be unenlightening to explicitly work out these directions without new ideas.

\section{Future work}

This section discusses possibilities for future work.

Question 8.1 (Significance of coordinates). How are the (asymmetric) z-coordinates related to other bijections (Anderson [5] for general cores; Ford-Mai-Sze [17] for selfconjugate cores; the poset formulation of Stanley-Zanello [27] developed further in [1], [4], and [3]; etc.), which are generally more symmetric in $s$ and $t$ ?

Question 8.2. Can the computations, especially for Fayers' conjectures (Theorems 1.3 and 1.4) be simplified along the lines of Johnson's weighted Ehrhart reciprocity methods in [21] for Armstrong's conjectures? Is there an 'exponential' version of Ehrhart reciprocity?

Remark 8.3. In Theorems 1.1 and 1.3 (and with some parity casework in Theorems 1.2 and 1.4) one can certainly use implicit variants of the generating function calculations (which, for Fayers' weighted sums, give an 'exponential' prototype of basic Ehrhart theory, but not necessarily reciprocity) to first show that the average size is a polynomial in $s$ of degree at most 2, and then give 3 easy-to-determine pieces of information to uniquely determine the quadratic. One piece of information could be Remark 5.1, i.e. that Fayers' weighted average is linear in $s$; taking $s=1$ or ' $s=0$ ' could be two other pieces of information. For Armstrong's un-weighted average, Johnson [21] uses the 3 pieces ' $s=0$ ', $s=1$, and $s=-t-1$ (using weighted Ehrhart reciprocity in the last case; one could also more concretely use the vanishing of $\left(\begin{array}{c}s+t-1 \\ t+*\end{array}\right)$ for $\left.* \geqslant 0\right)$. However, concrete explicit computations have their benefits, and implicit variants are already well-exposited in [21].

More concretely, one could study sums of higher powers of $|\lambda|$ or other statistics.

Question 8.4. For integers $e \geqslant 0$, what can one say about $\sum_{\lambda \in \mathcal{C}_{s} \cap \mathcal{C}_{t}}|\lambda|^{e}$, or the weighted sum $\sum_{\lambda \in \mathcal{C}_{s} \cap \mathcal{C}_{t}}\left|\operatorname{Stab}_{G_{s, t}}(\lambda)\right|^{-1} \cdot|\lambda|^{e}$ ? Can we study other statistics, such as length (number of nonzero parts)? Do these have a nice form? What do they say about the distribution of $(s, t)$-cores, indexed by size?

Remark 8.5. As Levent Alpoge points out, it may be more natural to look at moments (e.g. in the un-weighted case, essentially sums of powers of $|\lambda|-\frac{1}{24}(s-1)(t-1)(s+t+1)$ ). For specific conjectures of Ekhad and Zeilberger (supported by numerical evidence), see [10, First Challenge] (which was posted on the arXiv a few weeks after v3 of the present paper).

Remark 8.6. For any finite exponent $e$ these sums can in principle be computed explicitly by the cyclic shift methods used in Theorems 1.1 and 1.3. As a start, the generating function calculations show that both the un-weighted and weighted averages are polynomials in $s$ (but a priori only rational expressions in $t$ ) of degree at most $2 e$. Using the 
higher degree analog of Remark 5.1, one can reduce the degree bound to $2 e-1$ in the weighted case. In the un-weighted case, one can use the symmetry in $s, t$ to show that the un-weighted average is always a polynomial in $s, t$, hence in fact a symmetric polynomial.

Alternatively, one could ask about generating functions of $(s, t)$-cores. Note that the generating function for $s$-cores (indexed by size) has a well-known nontrivial expression, given for instance in [19, Bijection 1] (based on the $s$-core operation).

Question 8.7. Does the generating function for $(s, t)$-cores (indexed by size) have a nice form or any interesting properties?

Remark 8.8. Of course, if $s, t$ are coprime, this is a polynomial (as there are finitely many $(s, t)$-cores). See [22] for some potential progress by W. Keith in this direction. On the other hand, if $g:=\operatorname{gcd}(s, t)>1$, then [7] gives the $(s, t)$-generating function in terms of the $(s / g, t / g)$-generating function (thanks to Rishi Nath for pointing out this reference).

One could perhaps also ask further interesting questions about $t$-cores of different $s$-cores, following $[13,15]$. For example, are there unexplored natural definitions of "randomness"? Or is there anything one can do with the following remark?

Remark 8.9. The group $G_{s, t}$ (Definition 3.7) acts not only on $\mathcal{C}_{s}$ (via $s$-sets or beta-sets of $s$-cores), but on the whole set of partitions (via beta-sets). Similarly, the group $H_{s, t}$ (Definition 6.6) acts not only on $\mathcal{D}_{s}$, but also on $\mathcal{C}_{s}$ (via $s$-sets or beta-sets), and the whole set of partitions (via beta-sets).

In a different direction, one could investigate numerical semigroups containing $s, t$, which inject into $\mathcal{C}_{s} \cap \mathcal{C}_{t}$ (as mentioned in the introduction). Do the techniques for $(s, t)$ cores help?

\section{Acknowledgements}

This research was conducted at the University of Minnesota Duluth REU and was supported by NSF grant 1358695 and NSA grant H98230-13-1-0273. The author especially thanks Joe Gallian for suggesting the problem and carefully proofreading the paper, Melanie Wood for suggesting exactly what to emphasize or de-emphasize, and Amol Aggarwal, Levent Alpoge, Ben Gunby, Rishi Nath, and Paul Johnson for interesting discussions and other helpful comments on the manuscript. The author also thanks Rishi Nath for pointing out the papers [4] and [7], Tewodros Amdeberhan for suggesting how to elucidate the shortest path to the proof of Theorem 1.6, an anonymous expert for suggesting several helpful changes, and an anonymous referee for a thorough reading. The author would also like to thank Paul Johnson [21] and Matthew Fayers [13, 15] for their excellent exposition on core partitions, Matthias Beck and Sinai Robins [9] for their wonderful exposition on Ehrhart theory, and Jørn Olsson [24] for a counterexample showing that the $t$-core and $s$-core operations do not generally commute. Finally, the author thanks the Overleaf website for speeding up the writing process. 


\section{References}

[1] A. Aggarwal, Armstrong's conjecture for $(k, m k+1)$-core partitions, European J. Combin. 47 (2015), 54-67.

[2] T. Amdeberhan, M. Apagodu, and D. Zeilberger, Wilf's "snake oil" method proves an identity in the Motzkin triangle, preprint (2015), arXiv:1507.07660v1.

[3] A. Aggarwal, When does the set of $(a, b, c)$-core partitions have a unique maximal element?, Electronic J. Combin. 22(2) (2015), \#P2.31.

[4] T. Amdeberhan and E. Leven, Multi-cores, posets, and lattice paths, preprint (2015), arXiv: 1406.2250v3.

[5] J. Anderson, Partitions which are simultaneously $t_{1^{-}}$and $t_{2}$-core, Discrete Math. 248 (2002), 237-243.

[6] D. Armstrong, C. Hanusa, and B. Jones, Results and conjectures on simultaneous core partitions, European J. Combin. 41 (2014), 205-220.

[7] D. Aukerman, B. Kane, and L. Sze. On simultaneous $s$-cores/t-cores. Discrete Math. 309 (2009), 2712-2720.

[8] M. T. L. Bizley, Derivation of a new formula for the number of minimal lattice paths from $(0,0)$ to $(k m, k n)$ having just $t$ contacts with the line $m y=n x$ and having no points above this line; and a proof of Grossman's formula for the number of paths which may touch but do not rise above this line, J. Inst. Actuar. 80 (1954), 55-62.

[9] M. Beck and S. Robins, Computing the continuous discretely, Undergraduate Texts in Mathematics, Springer, New York (2007), http://math.sfsu.edu/beck/papers/ noprint.pdf.

[10] S. B. Ekhad and D. Zeilberger, Explicit expressions for the variance and higher moments of the size of a simultaneous core partition and its limiting distribution, preprint (2015), arXiv: 1508.07637v2.

[11] W. Chen, H. Huang, and L. Wang, Average size of a self-conjugate $(s, t)$-core, Proc. Amer. Math. Soc., electronically published on December 21, 2015, DOI: doi:10.1090/proc/12729 (to appear in print).

[12] R. Dijkgraaf and P. Sulkowski, Instantons on ALE spaces and orbifold partitions, J. High Energy Phys. (2008), no. 03, arXiv:0712.1427v2.

[13] M. Fayers, The $t$-core of an s-core, J. Combin. Theory Ser. A 118 (2011), 1525-1539.

[14] M. Fayers, A generalisation of core partitions, J. Combin. Theory Ser. A 127 (2014), $58-84$.

[15] M. Fayers, $(s, t)$-cores: a weighted version of Armstrongs conjecture, preprint (2015), arXiv: $1504.01681 \mathrm{v} 1$.

[16] S. Fishel, and M. Vazirani, A bijection between dominant Shi regions and core partitions, European J. Combin. 31 (2010), 2087-2101.

[17] B. Ford, H. Mai, and L. Sze, Self-conjugate simultaneous $p$ - and $q$-core partitions and blocks of $A_{n}$, J. Number Theory 129 (2009), 858-865. 
[18] French IMO delegation (unknown problem author), 2000 IMO Shortlist Problem C6, in: D. Djukić, V. Janković, I. Matić, and N. Petrović, The IMO Compendium: A Collection of Problems Suggested for The International Mathematical Olympiads: 1959-2009 Second Edition, Springer Science \& Business Media (2011), pp. 301, 666; and in: online thread (accessed June 2015) at http://artofproblemsolving.com/ community/c6h219750p1218952.

[19] F. Garvan, D. Kim, and D. Stanton, Cranks and t-cores, Invent. Math. 101(1) (1990), $1-17$.

[20] G. James, Some combinatorial results involving Young diagrams, Math. Proc. Cambridge Philos. Soc. 83 (1978), 1-10.

[21] P. Johnson, Lattice points and simultaneous core partitions, preprint (2015), arXiv: 1502.07934v2.

[22] W. Keith, The generating function of simultaneous $s / t$-cores, online beamer presentation (accessed June 2015), http://math. drexel . edu/ keith/CGFBeamer . pdf.

[23] A. Lascoux, Ordering the affine symmetric group, Algebraic combinatorics and applications (Gößweinstein, 1999), 219-231, Springer, Berlin, 2001.

[24] J. B. Olsson, A theorem on the cores of partitions, J. Combin. Theory Ser. A 116 (2009), 733-740.

[25] J. Olsson and D. Stanton, Block inclusions and cores of partitions, Aequationes Math. 74 (2007), 90-110.

[26] G. Robinson, On the modular representations of the symmetric group, Part IV, Canadian J. Math. 6 (1954), 486-497, http://cms . math.ca/10.4153/CJM-1954-052-9.

[27] R. Stanley and F. Zanello, The Catalan case of Armstrong's conjecture on simultaneous core partitions, SIAM J. Discrete Math. 29 (2015), no. 1, 658-666.

[28] J. Vandehey, Containment in $(s, t)$-core partitions, preprint (2008), arXiv:0809.2134.

[29] J. Y. X. Yang, M. X. X. Zhong, and R. D. P. Zhou, On the enumeration of $(s, s+$ 1, s+2)-core partitions, European J. Combin. 49 (2015), 203-217. 